\title{
Analyst
}

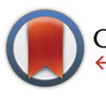

CrossMark

$\leftarrow$ click for updates

Cite this: Analyst, 2016, 141, 3126

\section{Functionalization of metal nanoclusters for biomedical applications}

\author{
Xiao-Rong Song, ${ }^{a, b}$ Nirmal Goswami, ${ }^{a}$ Huang-Hao Yang ${ }^{\star b}$ and Jianping Xie ${ }^{\star a}$
}

Metal nanoclusters (NCs) are emerging as a new class of functional nanomaterials in the area of biological sensing, labelling, imaging and therapy due to their unique physical and chemical properties, such as ultrasmall size, HOMO-LUMO transition, strong luminescence together with good photostability and biocompatibility. A recent surge of interest in this field is the surface functionalization of these metal NCs through which one can tailor their physicochemical properties, such as stability in solution, and strong luminescence, as well as their biodistribution and toxicity in biological systems, which in turn can empower these functionalized NCs with desirable targeting, imaging, and therapeutic ability for biomedical applications. In this review, we first introduce the functionalization strategies for the metal NCs developed in the past few years, followed by highlighting some biomedical applications of these functionalized metal NCs. We then discuss the difference of in vitro and in vivo fate as well as toxicity between various functionalized metal NCs. Finally, we present a short discussion on the current challenges and provide an outlook of the future developments of these functional metal NCs.

Received 2nd April 2016, Accepted 20th April 2016

DOI: 10.1039/c6an00773b www.rsc.org/analyst new class of functional materials. Due to their ultrasmall size approaching the Fermi-wavelength of electrons, metal NCs exhibit discrete energy levels and a multitude of molecular-like properties, $^{1,2}$ such as HOMO-LUMO transition, tunable luminescence, large Stokes shift, quantized charging, molecular chirality and magnetism. ${ }^{3,4}$ Recently, various kinds of metal NCs including gold $(\mathrm{Au})$, silver $(\mathrm{Ag})$ and copper $(\mathrm{Cu})$ NCs have been widely exploited for applications in the area of biological sensing, labelling, imaging and therapy. ${ }^{5,6}$ These metal NCs can produce multitudinous measurable signals, such as

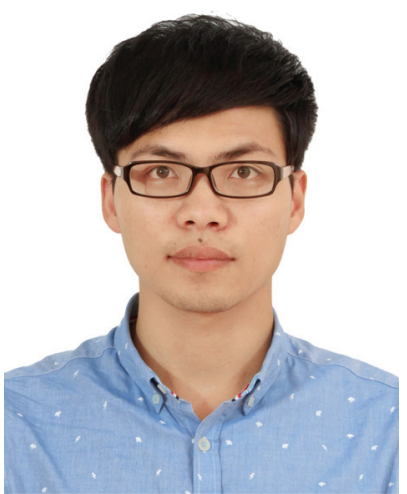

Xiao-Rong Song
Xiao-Rong Song received his BS (2012) from the College of Chemistry and Chemical Engineering at Fuzhou University (China). He continued to undertake his PhD study majoring in Analytical Chemistry in the Department of Chemistry at Fuzhou University under the supervision of Prof. Huang-Hao Yang, and currently he is an exchange student studying at the Department of Chemical \& Biomolecular Engineering, National University of Singapore, under the supervision of Prof. Jianping Xie. His research focuses on multifunctional nanomaterials, biosensors and nanomedicine.

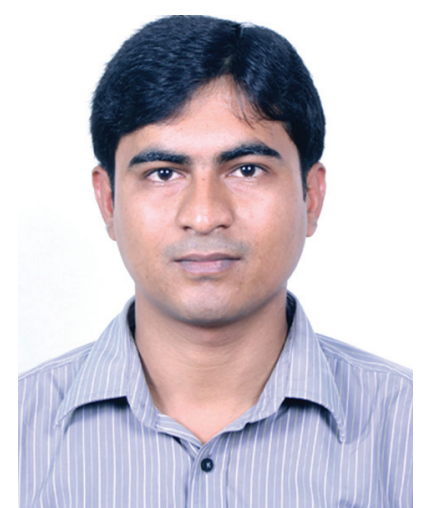

Nirmal Goswami
Nirmal Goswami is currently a postdoctoral fellow in Prof. Jianping Xie's group at the Department of Chemical and Biomolecular Engineering, National University of Singapore. He obtained his PhD (2014) in Chemistry from the S. N. Bose National Centre for Basic Sciences, India. He is interested in noble metal nanoclusters and their biomedical applications. 
luminescence and chemiluminescence, and have some distinctive features such as ultrafine size with narrow size distribution, and good photo-stability and biocompatibility. Because of all these unique attributes, metal NC based platforms have shown some superior performances over nanoparticles (NPs) in biomedical applications. ${ }^{7}$ For example, the use of large NPs may cause severe side effects in the body particularly in the liver and spleen when they are used in the in vivo set-up, since these NPs might not be able to escape from the kidney barrier. In contrast, the ultrafine size of metal NCs allows them to be efficiently cleared from the body without causing severe side effects.

In biomedical research, it is highly desired to develop reliable, sensitive, and biocompatible platforms. Sometimes, the materials need to maintain their intrinsic properties, such as stability, imaging, and therapeutic ability as well as targeting ability while facing the challenges of a complex matrix, cellular media or an in vivo environment, which are known to be consisting of a large number of proteins, thiols, amino acids, cells and other species or having a strong ionic strength. Therefore, it is extremely important to firmly control the surface chemistry of these materials since they play a major role in controlling many of the physicochemical properties of functional nanomaterials. Similar to other nanomaterials, the physicochemical properties of metal NCs could also be significantly influenced by the alteration of their surface chemistry. ${ }^{1}$ For example, the luminescence properties of Au NCs would not only be affected by the nature of the surface ligands, but also be susceptible to the environmental $\mathrm{pH}$ and temperature. ${ }^{8,9}$ The stability of these metal NCs could be further improved if the ligand shells are properly engineered on their surface. ${ }^{10-12}$ In fact, the ensuing research revealed the importance of functionalization of metal NCs to empower them with new biomedical functions as well as to finally improve their performance in applications including sensing, imaging, and therapeutics. ${ }^{7,13,14}$
While the synthesis, properties and biomedical applications of NCs have been published in some recent reviews, a concrete review related to their surface functionalization has not been summarized to date. ${ }^{5,13-15}$ Therefore, it would be of great importance to shed light on how to functionalize these metal NCs to fulfil the desired biomedical applications. The understanding of their functionalization properties can not only provide impactful insight into the surface properties of these metal NCs with respect to their interaction in the cellular medium but also can offer the basis to further design smart multifunctional metal NCs for practical applications. In line with this effort, this review will first focus on discussing the strategies related to the functionalization of metal NCs, followed by highlighting some biomedical applications of these functionalized NCs. Thereafter, we will summarize recent studies about the biodistribution and toxicity of metal NCs and then discuss the difference of the in vivo fate between various functionalized NCs. Finally, we will present a short discussion on the current challenges and give the outlook of the future developments of these functional metal NCs.

\section{Functionalization of metal NCs}

Benefiting from the contributions toward the developments of surface functionalization strategies, a number of methods have been established to either customize the metal NCs with necessary functional groups such as biomolecules and polymers, or to immobilize these metal NCs with a variety of inorganic nanostructures to further improve their performance in the biological set-up. In general, the surface functionalization of these metal NCs can be accomplished through two different ways: in situ functionalization during the synthesis and post-synthesis functionalization (after the synthesis). In

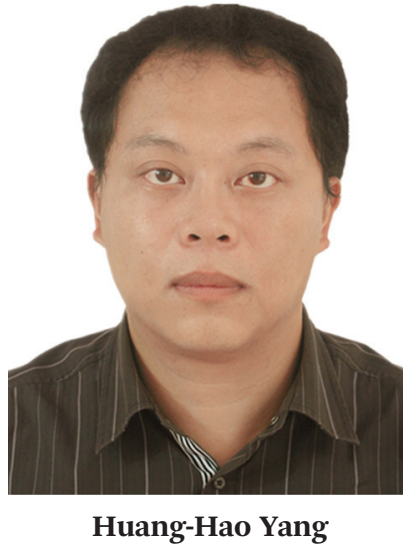

Huang-Hao Yang received his PhD from Xiamen University in 2002 and performed postdoctoral research in The Hong Kong University of Science and Technology from 2002 to 2004. He visited the University of Florida from 2007 to 2008 as a guest professor, and then joined Fuzhou University in 2008. His research interests mostly focus on biosensors, molecular diagnosis, nanomedicine, and cancer therapy. He has authored/coauthored over 150 peer-reviewed papers and total citations exceed 6300 (H-Index of 40). He was selected as a Changjiang Scholar Professor by Education Ministry of China in 2013 and became a fellow of the RSC in 2014.

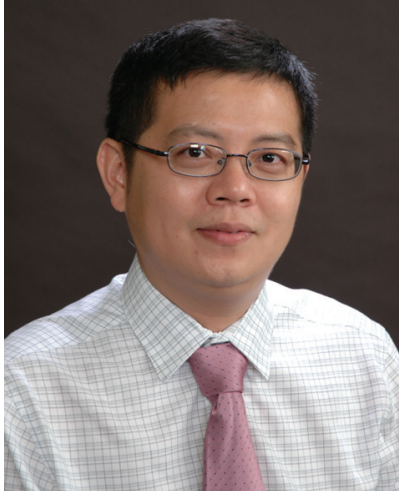

Jianping Xie

environmental applications index.html).
Jianping Xie received his $B S$ and MS degree from the Department of Chemical Engineering in Tsinghua University (China). He graduated with a PhD from the Singapore-MIT Alliance program. He joined the National University of Singapore as an Assistant Professor in 2010 and established the "Noble Metal Nanoclusters" research group. His major research interest is engineering ultrasmall metal nanoclusters for biomedical and (http://cheed.nus.edu.sg/stf/chexiej/ 
the following, we will discuss these two strategies by highlighting some examples.

\subsection{In situ functionalization during synthesis}

2.1.1 Thiols as protecting ligands. The use of thiols as surface protecting ligands can be traced back to the last century during the study of self-assembled monolayers on gold surfaces. ${ }^{16}$ It is now generally accepted that the strength of a thiolate-gold bond is close to that of a gold-gold bond, which means that thiolate ligands can modify the gold-gold bonding and result in the formation of a gold-sulfur interface. ${ }^{17}$ The robust covalent bond between gold and thiols first followed the exploitation of thiols as protecting ligands for the synthesis and functionalization of metal NPs and later made an impact on the pace of subsequent development of the thiolateprotected metal NCs (or thiolated NCs for short). In their early work, Whetten, Murray and other groups reported the successful synthesis and characterization of a number of thiolated $\mathrm{Au}$ NCs. ${ }^{18-20}$ Since then thiol-containing ligands remain as one of the most commonly used ligands for the synthesis, stabilization as well as the functionalization of metal NCs. ${ }^{8}$ The ease of availability as well as the rich surface chemistry of many thiol-containing compounds, including thiol-containing organosulfur compounds (e.g., mercaptobenzoic acid (MBA) and lipoic acid (LA)), thiol-containing peptides (e.g., tri-peptide, glutathione $(\mathrm{GSH})$ ), thiol-containing polymers (e.g., thiol terminated poly(ethylene glycol) (SH-PEG)), and thiol-containing amino acids (e.g., cysteine) have allowed researchers to use them as functional ligands for metal NCs with a potential for their use in many biomedical applications.

The strong bond between thiols and metal atoms as well as their facile formation has stimulated the accumulated achievements on the functionalization and applications of thiolated metal NCs. At the early stage, mono-thiolate ligands such as GSH were typically used as the ligand for the synthesis of metal NCs. However, advancement in the field by many researchers has led to the development of a number of metal NCs protected by various thiolate ligands. For instance, we have developed a unique $\mathrm{NaOH}$-mediated $\mathrm{NaBH}_{4}$-reduction method for producing various thiolated $\mathrm{Au}$ NCs. In fact, by using this method, additional surface functionalities could also be achieved by incorporating two different thiolate ligands into the NC surface (Fig. 1a). ${ }^{21}$ We found that the stability of thiolated Au NCs can be significantly improved by employing two thiol-terminated ligands having oppositely charged functional groups on the surface of the NCs. ${ }^{12}$ More recently, we also observed an intriguing optical absorption at about 780 and $980 \mathrm{~nm}$ of $\mathrm{Au}_{25}$ NCs protected by negatively charged thiolate ligands collocating with positively or neutrally charged thiolate ligands, which may be caused by the collocation of different charged ligands and surface charge anisotropy of thiolated Au NCs (Fig. 1b). ${ }^{22}$ These studies may open a new window in the investigation of the physicochemical properties of metal NCs and further facilitate their practical applications by designing their surface ligands.
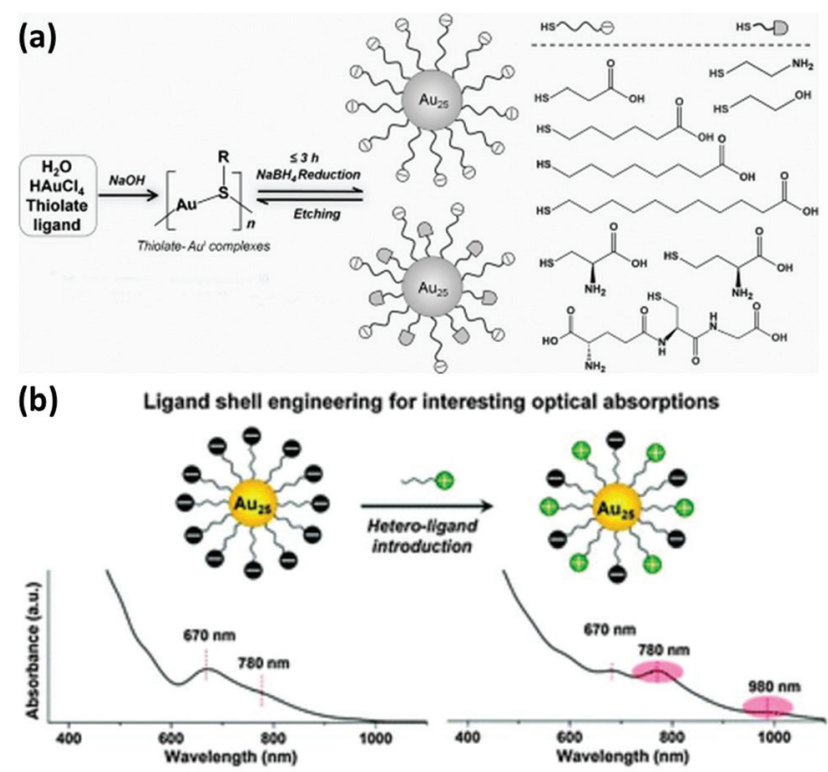

Fig. 1 (a) Schematic illustration of the $\mathrm{NaOH}$-mediated $\mathrm{NaBH}_{4}$ reduction method for the synthesis of thiolated Au NCs. Reprinted with permission from ref. 21. Copyright (2014) Wiley-VCH. (b) Schematic illustration and the intriguing optical absorption spectra of $\mathrm{Au}_{25} \mathrm{NCs}$ protected by negatively charged thiolate ligands collocating with positively charged thiolate ligands. Reprinted with permission from ref. 22. Copyright (2016) Royal Society of Chemistry.

In addition to the mono-thiolate ligands, di-thiolate ligands (i.e., a ligand containing two thiol groups) also gained significant attention for the functionalization of metal NCs due to their distinctive properties such as high stability and strong luminescence. For example, Au NCs functionalized with dithiolate ligand LA exhibited good long-term colloidal stability over a wide range of $\mathrm{pH}(2-13)$ and in the presence of high electrolyte concentration, making these LA-functionalized $\mathrm{Au}$ NCs promising for use in imaging and sensing applications. ${ }^{23}$ Similarly, dihydrolipoic acid (DHLA)-protected Ag NCs also showed high stability, which can be possibly ascribed to the bidentate chelating effect afforded by the di-thiolate group. ${ }^{24}$ Interestingly, the formation of the Au-di-thiolate bonding manner can also affect the physical properties of the NCs such as their optical properties. Wang and coworkers reported the synthesis of di-thiolate ligand 2,3-dimercaptopropane sulfonic (DMPS) protected $\mathrm{Au}_{4}$ clusters with high purity. ${ }^{25}$ Later, mixed di-thiolate durene and mono-thiolate phenylethanethiolate protected $\mathrm{Au}_{130}$ clusters synthesized by Wang's group was demonstrated to display multiple discrete absorption bands at about $355,490,584$, and $718 \mathrm{~nm}$, which may be attributed to the unique structural constraints imposed by the di-thiolate ligand on the interfacial bonding structures at the gold core surface. ${ }^{26}$ Despite the progress of di-thiolate-ligand-protected metal NCs, the studies in the field are still limited as compared to those studies in the field of mono-thiolated metal NCs, and therefore, more investigations should be carried out in future to provide more detailed and deeper information 
about their structures and properties in order to make them useful for practical applications. ${ }^{27,28}$

2.1.2 Functionalization with biomolecules. The functionalization of biomolecules on metal NCs is of crucial significance in chasing biocompatible platforms with minimal toxicity for biomedical applications. In the next section, we will discuss various biomolecule-based platforms such as nucleic acids, proteins, and peptides that have been used as templates for the synthesis and in situ functionalization of various metal NCs.

2.1.2.1 DNA oligonucleotides. DNA oligonucleotides have been used for a long time for the functionalization of various nanomaterials such as Au NPs and silica NPs. ${ }^{29}$ With the development of DNA-based architectures, studies on the binding between a metal ion/complex and DNA have also drawn much research interest. In 2004, Dickson and coworkers used DNA as templates for the synthesis of DNA-encapsulated $\mathrm{Ag}$ NCs (DNA-Ag NCs) due to the strong interaction between $\mathrm{Ag}^{+}$and DNA sequences prior to the reduction (Fig. 2a). ${ }^{30}$ Thereafter, more studies have been carried out to synthesize luminescent Ag NCs by using different DNA sequences, as well as to unravel the binding mechanism between DNA and the encapsulated Ag NCs. ${ }^{6}$ It has been reported that Ag NCs prefer to be functionalized with cytosine- or thymine-rich single stranded DNA due to the strong binding between $\operatorname{Ag}(\mathrm{I})$ and the $\mathrm{N}_{3}$ of cytosine or thymine. ${ }^{14}$ In fact, $\mathrm{Ag}$ NCs can be encapsulated in DNA with different structures, which include duplex, hairpin, G-quadruples and i-motif structures. ${ }^{6}$ DNA functionalization enables the Ag NCs to be well designed to fulfil different applications, such as incorporating the DNA tail with some aptamers to achieve specific binding and cell targeting. ${ }^{31}$

Motivated by the successful synthesis of DNA-Ag NCs, other metal NCs such as $\mathrm{Au}$ and $\mathrm{Cu}$ functionalized with DNA have also been reported. For example, Liu and coworkers demon-

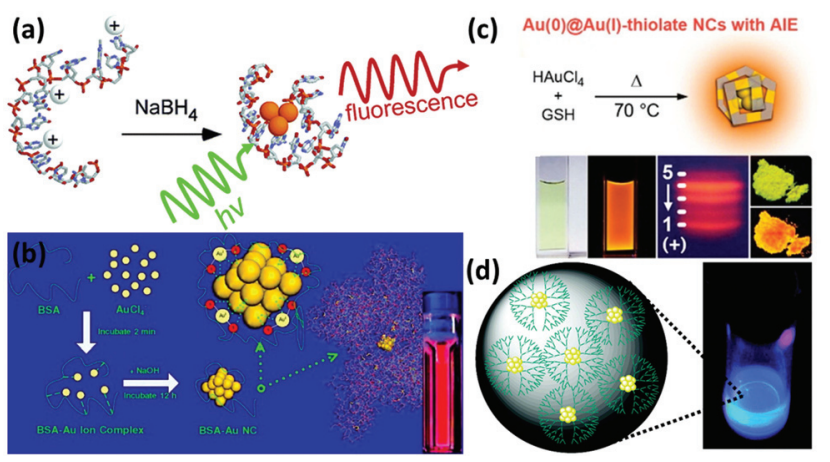

Fig. 2 (a) Schematic illustration of the formation of DNA-Ag NCs. Reprinted with permission from ref. 30. Copyright (2004) American Chemical Society. (b) Schematic illustration of the formation of Au NCs in BSA solution. Reprinted with permission from ref. 37. Copyright (2009) American Chemical Society. (c) Schematic illustration of the synthesis of highly luminescent $\mathrm{Au}$ NCs with aggregation-induced emission (AIE) properties. Reprinted with permission from ref. 50. Copyright (2012) American Chemical Society. (d) G4-OH PAMAM-encapsulated Au NCs and their luminescence under UV irradiation. Reprinted with permission from ref. 53. Copyright (2003) American Chemical Society. strated the preparation of DNA-encapsulated Au NCs with blue emission under mild reduction conditions. ${ }^{32}$ The formation of $\mathrm{Au}$ and $\mathrm{Cu}$ NCs is also highly dependent on the DNA sequences. $^{33}$ Interestingly, both double stranded DNA and poly-thymine single stranded DNA are proven to be able to protect the $\mathrm{Cu} \mathrm{NCs}^{34-36}$ Compared to $\mathrm{Ag}$ NCs that could be functionalized with ample DNA sequences, the functionalization of $\mathrm{Au}$ and $\mathrm{Cu}$ NCs with DNA still has a long way to go, especially for the exploitation of their physicochemical properties for practical applications.

2.1.2.2 Proteins and peptides. Proteins and peptides are biological macromolecules with an abundance of functional groups, such as carboxyl, thiol and amino groups. Some of these functional groups can be either used as reducing agents or served as protecting ligands/capping agents for metal NCs. For example, based on this unique property, we firstly reported the synthesis of protein-protected Au NCs by using bovine serum albumin (BSA) as a model protein (Fig. 2b). ${ }^{37}$ In this system, the protein layer on the NC surface could not only provide a number of outer functional groups (for their further functionalization if necessary), but also endow them with good biocompatibility in the biological set-up. The as-synthesized BSA-protected Au NCs (BSA-Au NCs) exhibited strong red emission with a high quantum yield (QY) of $\sim 6 \%$, and they also featured good stability in various media. Each of these properties made crucial contributions of protein-protected Au NCs in sensing, imaging and therapeutic applications. ${ }^{5,38,39}$ Following this work, various Au NCs have been synthesized and functionalized with different proteins, such as lysozyme, ${ }^{40}$ horseradish peroxidase, ${ }^{41}$ insulin, ${ }^{42}$ glucose oxidase, ${ }^{43}$ and ovalbumin. ${ }^{44}$ Some other metal NCs such as $\mathrm{Ag}$ and $\mathrm{Cu}$ have also been reported by using proteins as functionalizing ligands. ${ }^{45,46}$ Interestingly, the bioactivity of these proteins can be largely preserved in some cases, even after the synthesis of metal NCs. For example, the insulin-protected Au NCs (with red emission) can be used for fluorescence imaging, computed tomography (CT) imaging, as well as for in vivo blood-glucose regulation. ${ }^{42}$ The results strongly imply that the as-synthesized insulin-protected Au NCs largely retained the bioactivity as that of the commercial insulin. This is probably due to the ultrasmall size of Au NCs and the mild reaction conditions for the synthesis, which would not disturb the conformation and active sites of the protein.

Like protein, peptide is another class of attractive biomolecules that could be used to functionalize metal NCs. In particular, the amine and thiol groups of peptides could contribute to the protection and functionalization of metal NCs. Among the newly developed peptides for metal NCs, GSH is probably the most common choice for the preparation of metal NCs, including $\mathrm{Au}, \mathrm{Ag}$, Pt, and $\mathrm{Cu} \mathrm{NCs}{ }^{47-49}$ For example, we recently developed a facile synthesis strategy for the ultra-bright Au NCs (GSH-Au NCs, QY 15\%) by using $\mathrm{GSH}$ as both protecting and reducing agents under the reaction at $70{ }^{\circ} \mathrm{C}$ for $24 \mathrm{~h}$ (Fig. 2c). ${ }^{50}$ In some cases, long peptides can also be introduced to the surface of metal NCs. ${ }^{51}$ It should be noted that the designable sequence of peptides allows the synthesis of metal NCs with some unique properties, further 
endowing them with a certain charge and targeting capability for various applications, particularly in the biological set-up. ${ }^{52}$

2.1.2.3 Dendrimers and polymers. Dendrimers and polymers are also able to encapsulate and functionalize metal NCs. The presence of a myriad of functional groups within polymers and dendrimers makes them as promising templates for the facile synthesis of metal NCs with good water-solubility and high biocompatibility. For instance, poly(amidoamine) (PAMAM) dendrimers have been used as a scaffold for the synthesis and functionalization of $\mathrm{Au}$ (Fig. 2d) and $\mathrm{Ag}$ NCs. ${ }^{53,54}$ These NCs showed high stability in solution and exhibited high QY. In addition, their surface functionality can also be easily changed due to the availability of diverse generations of dendrimers. Another interesting fact is that these metal NCs are generally formed in the cavity of dendrimers, resulting in a highly charged outer surface. The dendrimer shell can provide a steric protection to improve the stability of metal NCs, and allow a further functionalization of these metal NCs through either a simple electrostatic interaction or a chemical conjugation for carrying the therapeutic agents. ${ }^{55}$ Apart from $\mathrm{Au}$ and $\mathrm{Ag}$ NCs, functionalized bimetallic NCs can also be obtained by using dendrimers as templates since dendrimers may possess robust metal chelating capability. For example, Au-Pd and Ag$\mathrm{Cu}$ NCs have been successfully encapsulated in PAMAM dendrimers. ${ }^{56,57}$ Similar to dendrimers, polymers with abundant functional groups are also proven to be competent agents for stabilizing metal NCs. Several synthetic methods such as photoreduction, chemical etching and microwave-assisted are reported for the preparation of polymer-encapsulated metal NCs. ${ }^{58-60}$ And a variety of polymers, including poly(ethylenimine) (PEI) ${ }^{61,62}$ and poly(vinylpyrrolidone) (PVP) ${ }^{63}$ have been largely used as protecting agents for the functionalization of metal NCs with various metal compositions. ${ }^{64}$

\subsection{Post-synthesis functionalization}

2.2.1 Bioconjugation. Bioconjugation is a powerful means to provide additional and useful functionalities on metal NCs. After the synthesis of metal NCs, a delicate selection of good stabilizers with desirable terminal functionalities, such as primary amine, carboxylic acid, and alcohol, is generally required to prevent the metal NCs from aggregation in solution and in a complicated biological environment. Started from the already formed metal NCs, it is sometimes necessary or beneficial to make further modifications with bio-related agents to broaden their applications in biomedical science. These bio-related agents have a wide spectrum of choices, ranging from small molecules (e.g., folic acid) to macromolecules (e.g., proteins and DNA) and genes. Conjugation chemistry mainly relies on the interaction between the terminal functional groups of the metal NCs and the functional groups of the desired biomolecules. One such post conjugation protocol is $\mathrm{N}$-(3-dimethylaminopropyl)- $\mathrm{N}$-ethyl carbodiimide (EDC) activated coupling between amine and carboxylic groups. For example, LA-protected Au NCs have been further functionalized with photosensitizers, namely protoporphyrin IX through the EDC and the $N$-hydroxysuccinimide (NHS)

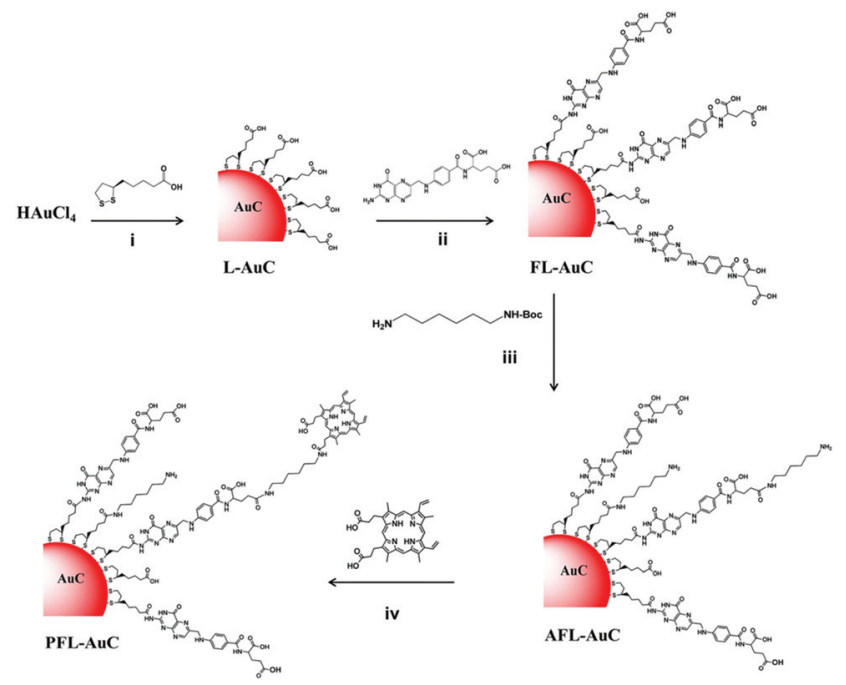

Fig. 3 Synthetic scheme of the photosensitizer-conjugated Au NCs. Reprinted with permission from ref. 65. Copyright (2015) American Chemical Society.

coupling reaction, which were designed for cancer treatment (Fig. 3). ${ }^{65}$ In addition to the small molecules, proteins and viruses are also effective to be integrated into functional metal NCs. For example, a model virus was recently functionalized on the surface of the as-designed water-soluble Au NCs. ${ }^{66}$ In particular, Au NCs with a carboxylic acid on the surface were first reacted with a six-carbon alcohol with terminal maleimide, resulting in the formation of maleimide-functionalized $\mathrm{Au}$ NCs. The as-functionalized Au NCs were further conjugated with a model virus, leading to the formation of virus-functionalized $\mathrm{Au}$ NCs. Over the past several years, bioconjugation of metal NCs has risen as one of the most popular strategies to functionalize the NC surfaces with desirable functionalities as the formation of irreversible bonds could largely benefit their practical applications. ${ }^{67}$

2.2.2 Post-synthesis ligand exchange. Post-synthesis ligand exchange is another efficient route for surface functionalization of metal NCs in solution. Ligand exchange reactions are known to be vital for the surface modification of quantum dots (QDs) and metal nanocrystals. ${ }^{68,69}$ In the NC research, the early work of Murray and coworkers ${ }^{70,71}$ has proven the ligand exchange reaction to be effective to functionalize the as-obtained metal NCs with new ligands and additional functionalities. ${ }^{72-75}$

Generally, the original ligands on metal NCs could be partially or completely exchanged by the incoming ligands depending on the NC species and the reaction conditions such as the concentration of NCs and the type of incoming ligand. ${ }^{76,77}$ In the case of partial ligand exchange, a selective functionality is incorporated into the NCs by exchanging some ligands on certain sites of NCs, without affecting the structures of the NCs. The complete exchange process, however, can replace the original monolayers with the desired functional groups along with a change of composition, structure, and properties of the resultant metal NCs in most cases. 
Functionalization of metal NCs by ligand exchange can further benefit their applications since the most stable NC species and desirable surface functionalities can be readily achieved in a delicate ligand exchange design. In addition, unlike the polydisperse chemical compositions in the products obtained from a typical direct synthesis method, the ligand exchange process might make possible the generation of NCs with a unique size distribution. ${ }^{74,78}$ For example, Tsukuda and coworkers carried out an interesting experiment, in which phosphine-stabilized $\mathrm{Au}_{11} \mathrm{NC}$ was the ligand exchanged with GSH in water (Fig. 4a). ${ }^{74}$ The resulting $\mathrm{Au}_{25}(\mathrm{SG})_{18}$ NCs were selectively produced in a large yield and high quality. Moreover, the new functionality imparted on metal NCs after the ligand exchange process can also be further modified with some specific agents for targeting purpose. ${ }^{79-81}$ As indicated above, the understanding of the ligand exchange process is of great importance for the functionalization and application of metal NCs.

2.2.3 Non-covalent interaction. A non-covalent interaction such as electrostatic interaction is also a good means to
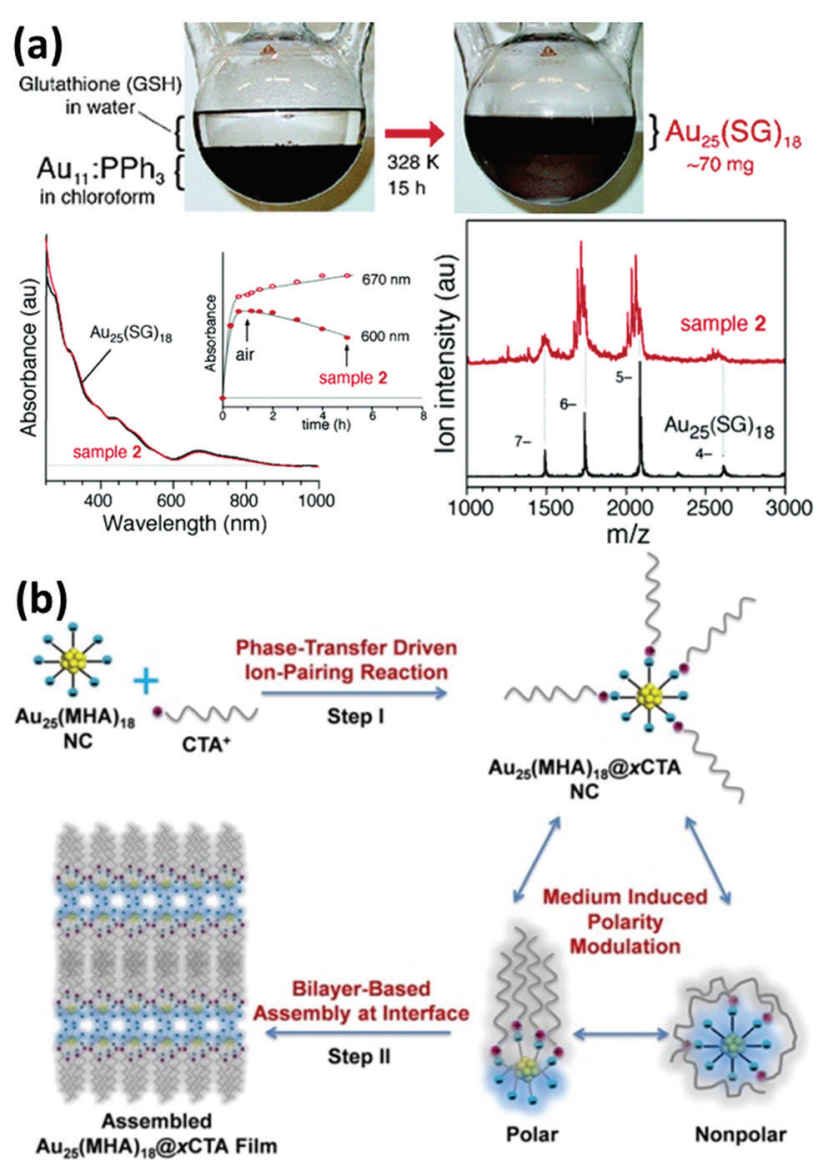

Fig. 4 (a) Schematic illustration and characterization of the ligand exchange reaction between phosphine-stabilized $\mathrm{Au}_{11} \mathrm{NCs}$ (in chloroform) and GSH in water. Reprinted with permission from ref. 74. Copyright (2005) American Chemical Society. (b) Schematic illustration of the synthesis of amphiphilic $\mathrm{Au}_{25}(\mathrm{MHA})_{18}$ @xCTA NCs by the phase-transfer driven ion-pairing reaction. Reprinted with permission from ref. 83 . Copyright (2015) American Chemical Society. further functionalize the as-synthesized metal NCs in solution. Generally, proteins with a certain charge can be functionalized on the surface of NPs and NCs carrying an opposite charge, through a direct electrostatic interaction. For example, Chang and coworkers have functionalized the 11-mercaptoundecanoic acid (MUA)-protected Au NCs with platelet derived growth factor AA (PDGF AA) after a simple incubation at room temperature. ${ }^{82}$ Recently, we have developed a simple surface modification method for the preparation of an amphiphilic metal NC (Fig. 4b). ${ }^{83}$ In particular, 6-mercaptohexanoic acid (MHA)-protected Au NCs with a certain number of anionic surface groups (carboxylate, $-\mathrm{COO}^{-}$) can be ion-paired with some hydrophobic cations (e.g., cetyl-trimethyl-ammonium, $\mathrm{CTA}^{+}$), generating amphiphilic Au NCs largely due to the coexistence of flexible hydrophilic MHA and hydrophobic MHA...CTA ligands in a comparable amount onto the NC surface. With the imparted amphiphilicity, the as-designed metal NCs may be attractive in the design of NC-based hybrid nanomaterials and self-assemblies, finding potential in both basic and applied researches. In another elegant study, Lee and coworkers demonstrated an effective post functionalization strategy to enhance the luminescence efficiencies of well-defined gold clusters. ${ }^{84}$ In particular, $\mathrm{Au}_{22}(\mathrm{SG})_{18}$ NCs functionalized with tetraoctylammonium (TOA) cations showed a luminescence QY greater than $60 \%$ due to the rigidified $\mathrm{Au}(\mathrm{I})$-thiolate shell caused by the significant intramolecular interaction between the alkyl chains of TOA cations.

\subsection{Metal NC-based nanocomposites}

The improvement of materials' performance can provide further opportunity to broaden their utility in practical applications. The influence of inorganic materials based nanocomposites on the physicochemical properties of metal NCs has received worldwide research interest owing to the possibility of generating multiple functions in a single entity. Compared with individual metal NCs, these hybrid nanocomposites may feature improved performance along with some new emerged properties resulting from the synergistic effects of metal NCs and other components. Some examples are discussed below.

2.3.1 Carbon-based nanomaterials. Carbon-based nanomaterials have been taken into account for the development of NC-based nanocomposites. Among the carbon-based materials, graphene and its water-soluble derivative, graphene oxide (GO), has been particularly considered as an important class of materials for the construction of NC-based hybrid nanocomposites with a desired function. Due to the presence of large specific surface areas and $\mathrm{sp}^{2}$-hybridized carbons in GO, recently, non-covalent fabrication has emerged as a promising method for developing such a hybrid nanocomposite of metal NCs and GO. ${ }^{85-87}$ This fabrication can be easily managed through the design of NC surface functionalities such as DNA and protein for integrating the NCs with GO. For example, Willner and coworkers synthesized the nanocomposites of DNA-Ag NCs and GO, which provide a versatile platform for the detection of genes and proteins, as well as the 


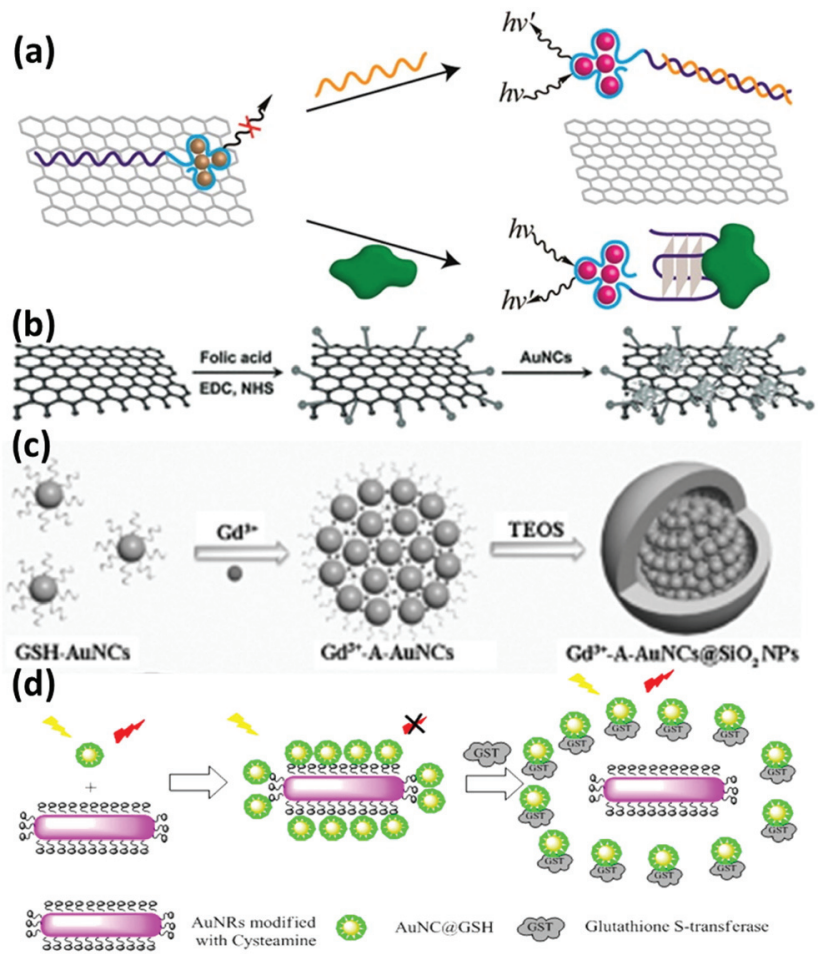

Fig. 5 Schematic illustration of the preparation of the nanocomposites of (a) DNA-Ag NCs/GO; reprinted with permission from ref. 88. Copyright (2013) American Chemical Society. (b) Lysozyme-Au NCs/GO; reprinted with permission from ref. 90. Copyright (2013) Wiley-VCH. (c) $\mathrm{Gd}^{3+}-\mathrm{A}-\mathrm{Au} \mathrm{NCs} \mathrm{SSiO}_{2} \mathrm{NPs}$; reprinted with permission from ref. 100. Copyright (2014) Wiley-VCH. (d) GSH-Au NCs and Au NRs based FRET system. Reprinted with permission from ref. 104. Copyright (2015) American Chemical Society.

analysis of aptamer-substrate complexes (Fig. 5a) ${ }^{88}$ In their study, the single stranded nucleic acids acted as templates to prepare Ag NCs, followed by their absorption by GO owing to the $\pi-\pi$ stacking interactions between hexagonal graphitic units of GO and nucleotides. Proteins could also facilitate the assembly of metal NCs and GO. For instance, Yan and coworkers used transferrin-functionalized Au NCs (Tf-Au NCs) and GO to form nanocomposites (Tf-Au NCs/GO) through the hydrogen bond and a hydrophobic interaction between $\mathrm{Tf}$ and GO. ${ }^{89}$ Due to the diversity of proteins, the surface charge of proteins can also contribute to the fabrication of nanocomposites of metal NCs and GO. Another good example is from $\mathrm{Qu}$ and coworkers. They used the lysozyme-stabilized $\mathrm{Au}$ NCs (lysozyme-Au NCs) to integrate with GO through the electrostatic interactions in which lysozyme is positively charged while GO is negatively charged at neutral $\mathrm{pH}$ (Fig. 5b). ${ }^{90}$ Alternatively, the easy synthesis process could also make possible the straightforward growth of metal NCs on the surface of GO. ${ }^{91-93}$ It is reported that the adsorption of metal salts on the preformed DNA/GO complexes, followed by the reduction of metal ions could construct Pt NCs and GO nanocomposites with a good environmental stability and durability. ${ }^{94}$ Apart from GO, carbon nanotubes (CNTs) were also widely used for fabricating NC-based nanocomposites. Various metal NCs, including $\mathrm{Ag}, \mathrm{Au}, \mathrm{Pt}$, and $\mathrm{Cu}$, have been functionalized with CNTs through multifarious strategies. ${ }^{95-97}$ For example, DNA-Ag NCs have been functionalized with CNTs to form CNT-Ag NC nanocomposites, which can be used as an efficient probe for an amplified detection of analytes. ${ }^{95}$

2.3.2 Silica coating. Silica coating is one of the most used strategies for the surface modification of nanomaterials for biological applications. ${ }^{98}$ NPs with a silica shell often exhibit good water-solubility and stability as well as a low nonspecific binding with the biological matrix. Different from metal NPs with a relatively larger size, metal NCs are very small and it is a great challenge to coat a silica shell on metal NCs in a wellcontrolled manner. ${ }^{99,100}$ Recently, Wang and Lin reported the $\mathrm{Gd}^{3+}$-induced aggregation of Au NCs, followed by the encapsulation of the resulting aggregated Au NCs within a silica shell, leading to the formation of $\mathrm{Gd}^{3+}-\mathrm{A}-\mathrm{Au} \mathrm{NCs} @ \mathrm{SiO}_{2} \mathrm{NPs}$; the asdesigned nanocomposites could serve as in vitro and in vivo multimodal probes for cancer cell imaging (Fig. 5c). ${ }^{100}$ In this system, the silica shell not only endows the nanocomposites with further surface modifications but also plays a crucial role in improving the photo and chemical stability of the Au NCs in solution. Similarly, some groups carried out the surface coating of silica on the protein-encapsulated Au NCs or the aggregated Au NCs. ${ }^{101-103}$ The as-designed silica coating has a number of traits, including good stability and ease of surface modifications with a variety of functional groups using silane chemistry and commercially available silane coupling agents.

2.3.3 Metal nanomaterials and metal oxides. Metal nanomaterials and metal oxides are another class of good candidates that could be integrated into metal NCs to form multifunctional NC-based nanocomposites. For example, GSH-Au NCs have been functionalized with amine-terminated Au nanorods (Au NRs) to form a fluorescence resonance energy transfer (FRET) system that can be applied for the detection of glutathione S-transferase (GST) (Fig. 5d). ${ }^{104}$ Notably, semiconductor-metal composites have been widely studied in the development of catalysts. It has been reported that reducing the size of metal NPs would improve the photo-induced charge separation and then enhance the catalytic activity of the nanocomposites. ${ }^{105}$ Recently, the semiconductor-metal NC composites have been increasingly recognised as good catalysts. ${ }^{106}$ More recently, GSH-Au NCs, being photosensitizers, were loaded into $\mathrm{TiO}_{2}$ films for efficient visible light-induced hydrogen generation in neutral water. ${ }^{107}$ Overall, the construction of NC-based nanocomposites pushes forward the study of multifunctional nano-platforms and further facilitates their applications.

\section{Biomedical applications}

The functionalization strategies discussed above could not only improve the properties of metal NCs, such as stability in solution and biological media, and biocompatibility, but also play a vital role in the development of sensors, imaging probes and theranostic agents. The abundant surface functionalities 
on metal NCs can be designed either during the synthesis or through the post-synthesis treatments, making these multifunctional metal NCs a promising platform for fulfilling the differently desired applications. Since several nice reviews covering the applications of metal NCs for biomedical science have already been contributed, we will focus our discussions on their biomedical applications (e.g., imaging and therapeutic application), particularly based upon the endowed functions of functionalized metal NCs in solution as discussed above.

\subsection{For special binding and targeting}

With the rapid development of nanotechnology, nanomaterials based platforms are emerging as effective tools for highly specific and sensitive analysis of biological agents. Due to high QYs, good photostability, and biocompatibility, metal NCs have been widely used as signal reporters for biological analysis. However, a specific recognition in the complex biological environment still remains a great challenge since such a complexity can affect the detection limit and even sometimes induce a falsified positive result. To make these metal NCs more effective, their surfaces are often functionalized by means of biocompatible molecules.

Oligonucleotide is one such biomolecule that has been used for surface functionalization of these metal NCs. DNA-Ag NCs, particularly, have drawn much attention in the specific analysis and targeted imaging because of the simple synthesis of these NCs. To functionalize these NCs with recognizable elements, DNA aptamer sequences were often designed in the tail of a particular DNA template to achieve specific binding to various targets. ${ }^{108,109}$ Among them, an intriguing phenomenon of luminescence enhancement induced by the proximity of the G-rich overhang to $\mathrm{Ag}$ NCs has been exploited to develop the various target responsive sensors and imaging probes (Fig. 6a \& b). ${ }^{110,111}$ Moreover, the easy coupling between template DNA and luminescence quenchers, such as organic dyes and nanomaterials, facilitates the construction of aptamer-functionalized NC-based platforms for targeted imaging. ${ }^{112,113}$

Apart from the oligonucleotides, proteins and peptide functionalized metal NCs have also made important contributions to specific binding and targeting. ${ }^{114}$ One of the advantages of protein-functionalized metal NCs is that some proteins can directly target the cancer cells and also provide a platform for further functionalization with other specific agents for targeting. For example, Irudayaraj and coworkers reported the conjugation of BSA-Au NCs with Herceptin (Her-Au NCs), which can achieve specific targeting and nuclear localization of the asdesigned Au NCs in ErbB2 over-expressing breast cancer cells (Fig. 7). ${ }^{115}$ Notably, Her-Au NCs retained both the luminescence properties and the targeting capability. It was interesting that the as-designed Her-Au NCs exhibited an endosomal escape behaviour and targeted at nucleus of the cells with high efficiency, while the Alexa647 dye-labelled Herceptin primarily localized on the cell membranes since the free Herceptin has very limited ability to pass through the nuclear pore complex, indicating that functionalization of the target agents on Au NCs can in turn improve the targeting capability of the tar-

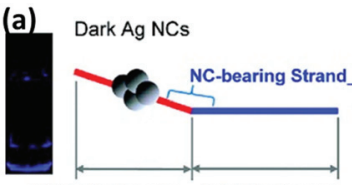

NC-nucleation seq. Hybridization seq

(b)

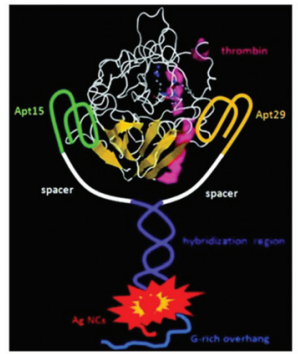

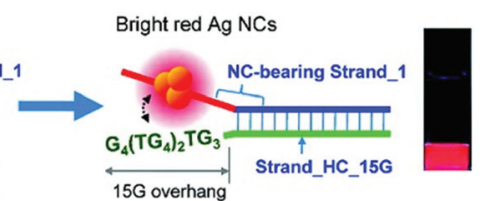

(c)

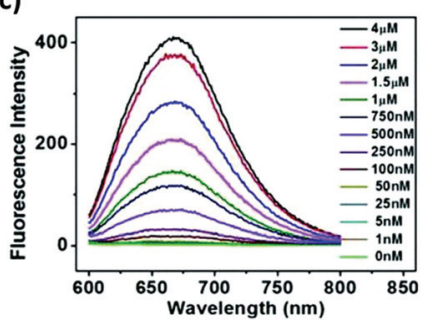

Fig. 6 (a) Schematic illustration showing the red emission enhancement of DNA-Ag NCs caused by the guanine proximity. Reprinted with permission from ref. 110. Copyright (2010) American Chemical Society. (b) Schematic illustration of the proximity of G-rich overhang to Ag NCs induced by the binding of two aptamers with thrombin, and (c) fluorescence spectra of $\mathrm{Ag} \mathrm{NCs}$ in the presence of different concentrations of thrombin. Reprinted with permission from ref. 111. Copyright (2012) American Chemical Society.

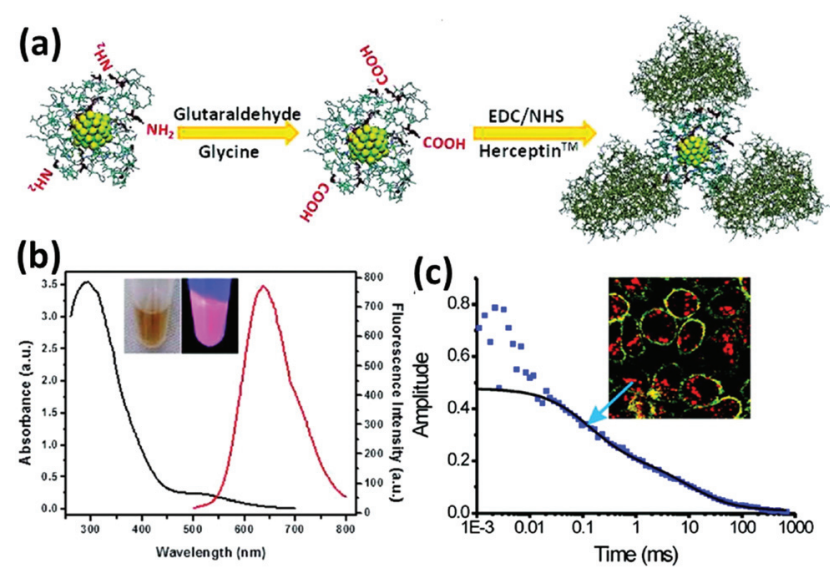

Fig. 7 (a) Schematic illustration of the preparation of Her-Au NCs. (b) UV-vis absorption and luminescence spectra of Au NCs. (c) Typical autocorrelation curve (solid squares) of Her-Au NCs diffusing inside the nucleus fitted with two components (black solid line). Inset is the image of SK-BR3 cells incubated with Her-Au NC-Alexa647 for $4 \mathrm{~h}$. Reprinted with permission from ref. 115. Copyright (2011) American Chemical Society.

geting agents. Likewise, the targetable Au NCs can be obtained by conjugating BSA-Au NCs with other agents, such as folic acid and TAT peptide, which can selectively bind with specific receptor expressing cancer cells and tumor tissues. ${ }^{116-120}$

\subsection{For multi-modal imaging}

Over the past few years, unprecedented efforts have been undertaken to improve the resolution and sensitivity of the bioimaging techniques, ranging from the traditional computed tomography (CT), ultrasound (US) imaging, and mag- 
netic resonance imaging (MRI), to emerging optical fluorescence imaging (FL), positron emission tomography (PET), and photoacoustic imaging (PAI). With the purpose of conquering the drawbacks of a single modal imaging and ultimately improving the diagnostic accuracy, many multimodalimaging platforms have been developed by integrating different imaging agents into a single system. In the following, we will discuss some examples in the pursuit of metal NCbased multimodal imaging platforms.

Among the various available medical imaging platforms, CT is one of the most frequently used hospital diagnostic tools with cost-effectiveness. Because of the higher atomic number and electron density of gold compared to conventionally used materials such as iodine, Au NPs have been proven to be a class of effective CT contrast agents. Since Au NCs intrinsically possess both FL and CT imaging capability, recent efforts have been geared up for designing Au NC-based multimodal imaging agents. For example, Cui and coworkers demonstrated a simple synthesis of folic acid conjugated GSH-Au NCs, which not only possess the targeting ability but also provide a versatile platform for FL and CT imaging (Fig. 8a). ${ }^{121}$ Such a dualmodal imaging probe can also be achieved by utilizing other luminescent Au NCs, such as insulin-protected Au NCs. ${ }^{42}$ For example, Zheng and coworkers reported the study of in vivo renal clearance properties of ultrasmall Au NPs by using FL and CT imaging. ${ }^{122}$

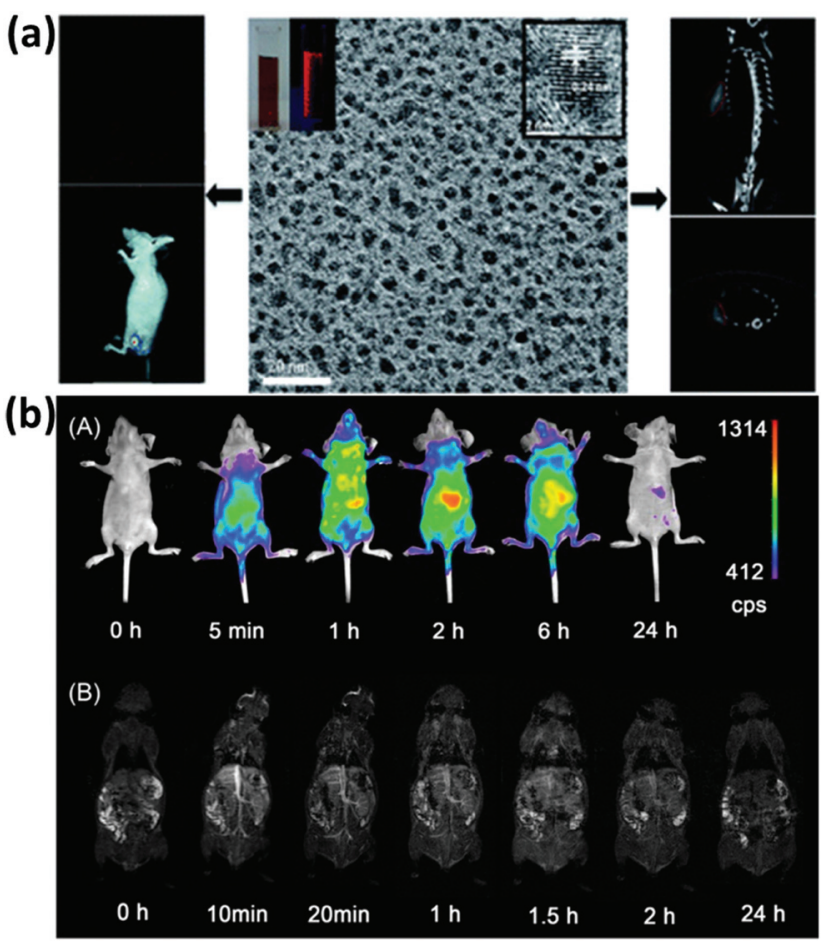

Fig. 8 (a) Dual-modal FL/CT imaging of gastric cancer using folic acid conjugated GSH-Au NCs. Reprinted with permission from ref. 121. Copyright (2013) Royal Society of Chemistry. (b) (A) NIR FL and (B) MR blood in vivo imaging using $\mathrm{BSA}-\mathrm{Gd}_{2} \mathrm{O}_{3} / \mathrm{Au}$ nanoprobes. Reprinted with permission from ref. 38. Copyright (2013) American Chemical Society.
To construct a reliable multimodal imaging probe, MRI has drawn much attention owing to its fine spatial resolution. ${ }^{123,124}$ In general, the combination of MRI contrast agents with $\mathrm{Au}$ NCs can be achieved through coupling Au NCs with paramagnetic agents or integrating Au NCs into magnetic NPs to form hybrid materials. Starting with the synthesis of $\mathrm{Au}$ NCs protected by proteins, Cai and coworkers reported the functionalization of $\mathrm{Gd}^{3+}$ on the surface of $\mathrm{Au}$ NCs through an electrostatic interaction to achieve FL/MRI dual-modal imaging. ${ }^{125}$ Similar tri-modal imaging probes were constructed by Kong and coworkers by functionalizing the peptide-protected $\mathrm{Au}$ NCs with $\mathrm{Gd}^{3+} \cdot{ }^{126}$ The as-prepared hybrid Au NCs retained good stability, good biocompatibility, and low toxicity, and no obvious $\mathrm{Gd}^{3+}$ leaching was observed in different solutions. In an alternative strategy, Yan and coworkers used BSA as a template for an in situ synthesis of a multifunctional $\mathrm{Gd}_{2} \mathrm{O}_{3} / \mathrm{Au}$ hybrid probe, which enables a successful near-infrared (NIR) FL and MR blood pool imaging attributing to their long blood circulation time (Fig. 8b). ${ }^{38}$ Further modification of the probe with arginine-glycine-aspartic acid peptide c(RGDyK) (RGD) also enabled the nanoprobe for in vivo targeted tumor imaging. Very recently, GSH-Au NCs were also functionalized with magnetic iron oxides through an electrostatic interaction to form a composite material for FL and MRI based dual-modal imaging. ${ }^{127}$

Apart from the above-mentioned methods, Au NCs have also been functionalized with PET contrast agents to achieve the goal of multi-modal imaging. For example, $\mathrm{Wu}$ and coworkers described a novel strategy for the synthesis of ${ }^{64} \mathrm{Cu}$ decorated $\mathrm{Au}$ NCs through the reduction of ${ }^{64} \mathrm{CuCl}$ followed by decoration on the surface of Au NCs. ${ }^{128}$ The as-synthesized ${ }^{64} \mathrm{Cu}$-decorated $\mathrm{Au}$ NCs can serve as self-illuminating NCs for dual-modal PET and NIR FL imaging based on Cerenkov resonance energy transfer (CRET). Other kinds of NCs, such as Ag and $\mathrm{Cu}$ NCs have also attracted much research interest in multi-modal imaging since $\mathrm{Ag}$ and $\mathrm{Cu}$ NCs both can be luminescent, and the radioactive $\left[{ }^{64} \mathrm{Cu}\right] \mathrm{Cu}$ NCs can be intrinsically used for PET imaging. ${ }^{129,130}$ Due to the diverse metal NCs and the rich functionalization strategies for designing functional NCs, the developments of NC-based multifunctional contrast agents are continuously becoming popular with time.

\subsection{For therapeutic application}

Functionalized nanomaterials have the potential to revolutionize the diagnosis and treatment of many diseases, including cancer which still remains as a major cause of human death. The development of therapeutic nanocomposite agents offers a new hope to elucidate drug resistance mechanisms and improve treatment outcomes. There have been many efforts to employ functionalized Au NCs for the construction of therapeutic platforms. In the following section, we will highlight some examples in details.

3.3.1 Radiotherapy (RT). Radiotherapy is one of the most effective treatments following surgical removal of tumors. However, a major challenge in RT is that the ionizing radiation cannot differentiate between the healthy tissues and solid 
tumors. ${ }^{131}$ Therefore, a high dose of radiation often kills the healthy cells. One efficient way to overcome this problem is to use radiosensitizers, and localize them in the tumor cells which could make tumor cells more vulnerable under radiation. It is now well-recognized that elements with a higher atomic number $(Z)$ will be a better radiosensitizer with improved RT effects. Compared to the commonly used radiosensitizers such as carbon $(Z=6)$, iodine $(Z=53)$, gadolinium $(Z=64)$ and platinum $(Z=78)$, gold has a larger atomic number $(Z=79)$, therefore, it should have a stronger radiation enhancement effect. However, Au NPs with particle sizes above $50 \mathrm{~nm}$ were reported to be trapped by the reticuloendothelial system (RES) organs, which could result in low tumor uptake. Although the Au NPs with a size below $20 \mathrm{~nm}$ would escape RES absorption, a long term potential toxicity still remains since they may not be efficiently eliminated from the body. ${ }^{132,133}$ Due to the small size and low toxicity of Au NCs, recently our group used the $\mathrm{GSH}$-protected $\mathrm{Au}_{25}$ NCs as radiosensitizers for cancer RT. The as-designed Au NC-based radiosensitizers have shown attractive features such as high tumor accumulation, strong radiation enhancement, good renal clearance, and low toxicity in the in vivo set-up (Fig. 9). ${ }^{134}$ of note, the GSH-Au ${ }_{25}$ NCs showed better accumulation in tumor cells, and therefore show a stronger enhancement for cancer $\mathrm{RT}$ as compared to that of $\mathrm{BSA}-\mathrm{Au}_{25} \mathrm{NCs}$ in both in vitro and in vivo set-ups. In a more recent study, $\mathrm{Au}_{10-12} \mathrm{SG}_{10-12}$ and $\mathrm{Au}_{29-43} \mathrm{SG}_{27-37}$ NCs were also developed as efficient radiosensitizers for cancer RT, showing significant RT enhancement. ${ }^{132,133}$ It should be noted that some differences in biodistribution of Au NCs still can be observed between these three kinds of Au NCs. The data highlight that the composition and surface functionalization of Au NCs can affect their

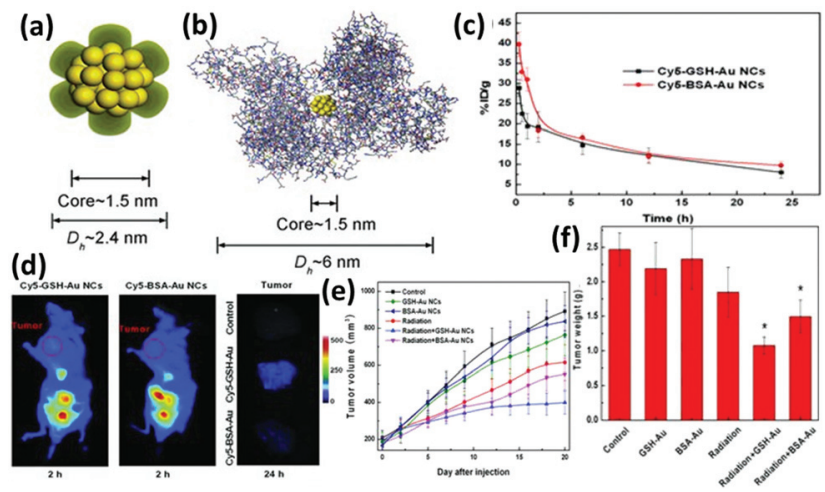

Fig. 9 Schematic illustration of the core-shell structure of (a) $\mathrm{GSH}-\mathrm{Au}_{25} \mathrm{NCs}$ and (b) $\mathrm{BSA}-\mathrm{Au}_{25} \mathrm{NCs}$. (c) In vivo pharmacokinetics studies of GSH-Au $u_{25} \mathrm{NCs}$ (black line) and $\mathrm{BSA}-\mathrm{Au}_{25} \mathrm{NCs}$ (red line). (d) Fluorescence images of mice treated with GSH-Au 25 NCs (left panel) and $\mathrm{BSA}-\mathrm{Au}_{25} \mathrm{NCs}$ (middle panel) at $2 \mathrm{~h}$ after injection; the right panel is the tumor images (false colour) at $24 \mathrm{~h}$ after injection of the control group (top), $\mathrm{GSH}-\mathrm{Au}_{25} \mathrm{NC}$ group (middle), and $\mathrm{BSA}-\mathrm{Au}_{25} \mathrm{NC}$ group (down). Time-course studies of tumor (e) volumes and ( $f$ ) weights of mice treated with GSH- and $\mathrm{BSA}-\mathrm{Au}_{25} \mathrm{NCs}$ at a concentration of $10 \mathrm{mg}$ $\mathrm{Au}$ per kg body. Reprinted with permission from ref. 134. Copyright (2014) Wiley-VCH. biodistribution and eventually determine their efficacy as radiosensitizers in cancer RT.

3.3.2 Photodynamic therapy (PDT). Photodynamic therapy, a clinically approved non-invasive therapeutic strategy, is also an alternative therapeutic method exploited for metal NCs. ${ }^{135}$ In PDT, the photosensitizers should have capability in generating reactive oxygen species (ROS) when they are irradiated with an appropriate light source. Therefore, when they are localizing at the targeted area, a selective destruction of that targeted area can be achieved by PDT from ROS generated by the photosensitizers. With the recognition that several functionalized $\mathrm{Au}$ NCs, such as Au NCs embedded in a polymer film, BSA-Au NCs, and thiolated $\mathrm{Au}$ NCs, can generate singlet oxygen species $\left({ }^{1} \mathrm{O}_{2}\right),{ }^{136-138}$ researchers have started exploiting this class of materials as photosensitizers for PDT. For example, Jin and coworkers demonstrated that ${ }^{1} \mathrm{O}_{2}$ can be efficiently produced by a direct photosensitization of captopril/phenylethanethiol-protected $\mathrm{Au}_{25}$ NCs under visible/NIR light irradiation $(532,650$, and $808 \mathrm{~nm}) .{ }^{139}$ Based on this unique observation, they further used the captopril-protected $\mathrm{Au}_{25}$ NCs as biocompatible photosensitizers for PDT combating cancer cells. The advantage of using Au NCs as photosensitizers over traditional organic photosensitizers can also be evident from their tumor targeting capability. In a separate study, Hwang and coworkers developed a theranostic platform based on a nucleus-targeting Au NC (TAT peptide-Au NC) (Fig. 10). ${ }^{120}$ The as-prepared TAT peptide-Au NCs can generate ${ }^{1} \mathrm{O}_{2}$ under the irradiation of $980 \mathrm{~nm}$ light, from a direct observation of phosphorescence emission at $1261 \mathrm{~nm}$ with a QY of 0.046. The as-designed peptide-Au NCs have been used for the nucleus targeted PDT.

Apart from their use as therapeutic agents, metal NCs can also serve as carriers to deliver biomedical agents such as
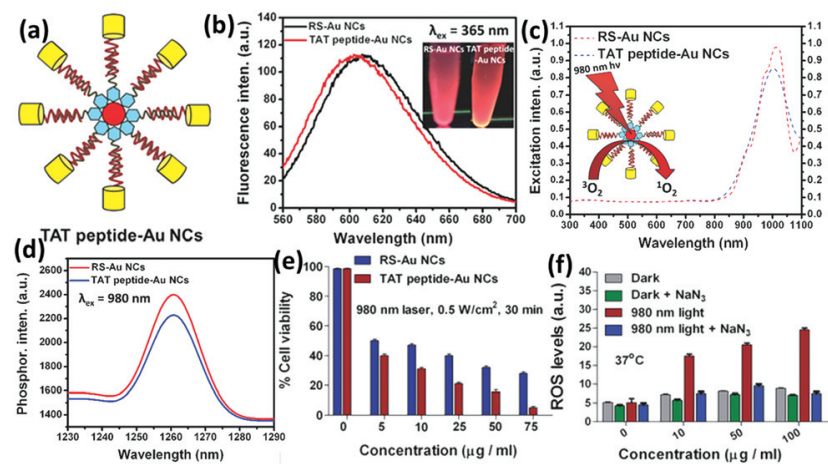

Fig. 10 (a) Schematic illustration of the nucleus-targeting TAT peptideAu NCs. (b) Photoemission spectrum of alkyl thiolated Au NCs (RS-Au NCs) and TAT peptide-Au NCs. (c) Photoexcitation spectrum of singlet oxygen phosphorescence of RS-Au NCs and TAT peptide-Au NCs $\left(\lambda_{\mathrm{em}}=\right.$ $1261 \mathrm{~nm}$ ). (d) Singlet oxygen photoemission spectra of RS-Au NCs and TAT peptide-Au NCs at $980 \mathrm{~nm}$ (excitation wavelength). (e) Cell viability of RS-Au NCs and TAT peptide-Au NCs internalized HeLa cells under photo-irradiation conditions at $37^{\circ} \mathrm{C}$. (f) ROS generation monitored by the mean fluorescence using flow cytometry for TAT peptide-Au NC internalized HeLa cells followed by photo-irradiation with and without $\mathrm{NaN}_{3}$ pre-treatment. Reprinted with permission from ref. 120. Copyright (2015) Wiley-VCH. 
drugs (e.g., chemotherapeutics and photosensitizers) and genes. Therapeutic targeted delivery vehicles have made a great contribution in the fight against cancers and often showed a better performance than a single therapeutic agent which often suffers from the lack of targeting, limited tumor uptake, and sometimes severe side effects. ${ }^{140-142}$ Among the most commonly used nanomaterials to deliver drugs into the desired sections, metal NCs show some merits owing to their ultrasmall size, rich surface functionalities, low toxicity, and intrinsic strong luminescence, which could be used to develop a safe and efficient platform for imaging-guided therapy. Generally, the therapeutic agents can be functionalized on metal NCs either by a direct electrostatic interaction or by a chemical conjugation. In terms of electrostatic interactions, polymers are mainly used as linkers to bind with NCs and therapeutic agents for the formation of nanocarriers. ${ }^{143-147}$ Notably, these NC-based therapeutic vehicles can achieve a stimuli responsive delivery to reduce the systematic toxicity. In terms of chemical conjugation, thiols and proteins play an important role for the protection of metal NCs and they could be readily conjugated with a certain therapeutic agent. ${ }^{65,148}$ The metal NC-based drug delivery vehicles show great potential as a tumor-targeted drug delivery carrier and they could further act as a promising theranostic platform for multi-modal therapy and imagingguided therapy that could improve the treatment outcome.

\section{Biodistribution and toxicity}

With the development of nanotechnology in biomedical research and clinical science, it is greatly desired to understand the influence of nanomaterials on human health. In order to evaluate the risks caused by the nanomaterials, plenty of efforts have been made to delineate the cellular uptake, cytotoxicity, biodistribution, as well as long term toxicity of nanomaterials. To date, Au nanomaterials are one of the most popular model materials in the systematic investigations of toxicity in biological systems. However, there are some inconsistencies existing due to the difference in the synthetic process, surface chemistry, cell lines, and evaluated methods. In contrast to the nanomaterials (or NPs) with a relatively larger size, ultrasmall metal NCs with well-defined size and structure have provided some new insights into the safety evaluation due to their attractive feature of small size with narrow size distribution, rich surface chemistry, and intriguing physical and chemical properties, allowing us to understand the related information at molecular and atomic levels.

\subsection{Cellular uptake and cytotoxicity}

The evaluation of toxicity would be relatively easier in the case of metal NCs as compared to the NP system due to the fact that the narrow size distribution of NCs would allow researchers to slip the size dependent toxicity that always needs to be considered in the studies of NPs. As an emerging luminescent probe for in vitro imaging and targeting, Au NCs show extremely low cytotoxicity on various cell lines. In order to systemi- cally study the cytotoxicity of these Au NCs, it is critical to understand their cellular uptake mechanism and to study the interaction between $\mathrm{Au}$ NCs with the intracellular environments. Significantly, some recent studies emphasized the importance of surface functionalization towards their uptake manner and cytotoxicity. ${ }^{149}$ For example, Nienhaus and coworkers studied the cellular uptake mechanism by using LAprotected Au NCs and HeLa cells. ${ }^{150}$ It was demonstrated that Au NCs can enter cells through pinocytosis involving multiple mechanisms, making Au NCs reside firstly in endosome and later in lysosome. However, some differences can be seen when these $\mathrm{Au}$ NCs were conjugated with targeting agents such as Herceptin to form Her-Au NCs, which could escape the endo-lysosomal pathway and enter the nucleus of cancer cells. ${ }^{115,151}$ The difference in the uptake behaviour would also affect their cytotoxicity by generating toxic intracellular species such as ROS. Recently, we also described the different cellular internalization between Au NCs functionalized with different ligands. ${ }^{11}$ It was observed that intracellular ROS was higher in cells exposed to mercaptopropionic acid (MPA)-protected Au NCs than that in the case of GSH-Au NCs. However, this effect did not induce any detrimental cellular effect, and instead, it enhanced the cell metabolism leading to a higher cell proliferation. In another example, Tao and coworkers systematically studied the BSA-Au NCs and found that Au NCs induced the decline of cell viability to varying degrees in a cell-, dose- and time-dependent manner. ${ }^{152}$ More recently, Guével and coworkers used human derived-monocyte dendritic cells (DCs) as a model to investigate the intracellular accumulation and immune response of $\mathrm{Au}$ NCs functionalized with zwitterionic ligands and PEG. ${ }^{153}$ Their results revealed that zwitterionic ligand functionalized Au NCs showed better performance than the PEG-functionalized Au NCs, exhibiting low cytotoxicity and a strong immunosuppressive response, which may make the as-functionalized Au NCs a novel vaccine. Even though there is no consensus yet about the cytotoxicity of Au NCs, the surface chemistry of $\mathrm{Au}$ NCs does play pivotal roles in affecting the cellular uptake manner and cytotoxicity.

\subsection{In vivo biodistribution and clearance}

A clinical acceptable agent approved by the US Food and Drug Administration (FDA) should be effectively cleared from the body after injection in a reasonable time period. ${ }^{154-156}$ During the past few years, some NPs with a hydrodynamic diameter (HD) below $5.5 \mathrm{~nm}$ have shown effective and rapid clearance through kidney. ${ }^{154,157-159} \mathrm{Au}$ NCs also have drawn much attention due to their small size and multifunctional applications in biomedicine. ${ }^{134,160-162}$ However, the surface chemistry would affect their biodistribution and clearance. ${ }^{163}$ The prime consideration is whether the size of functionalized Au NCs can satisfy the kidney filtration threshold $(\sim 5.5 \mathrm{~nm})$. It is worthy to be mentioned that some zwitterionic ligands (e.g., GSH), proteins (e.g., BSA), and PEG are the mainly used surface functionalization of Au NCs. Due to the relatively large size of proteins, protein-functionalized Au NCs would face a great challenge to be renally cleared. For example, Zhang and co- 


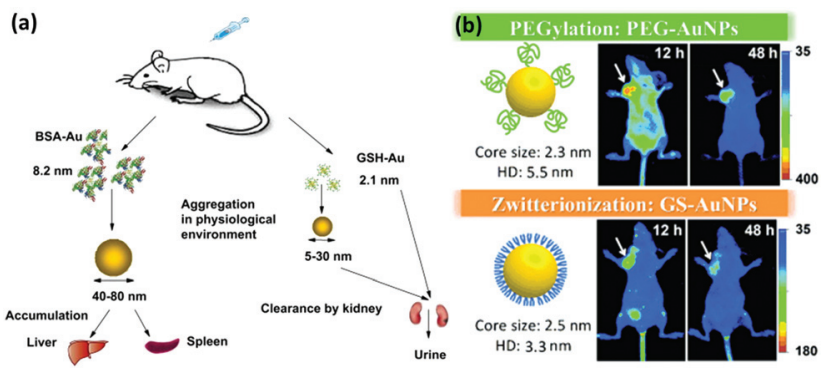

Fig. 11 (a) Outline of the biodistribution and renal clearance the GSHand $\mathrm{BSA}-\mathrm{Au}_{25} \mathrm{NCs}$. Reprinted with permission from ref. 160. Copyright (2012) Elsevier. (b) PEGylation and zwitterionization have distinct effects on the pharmacokinetic and tumor-targeting properties of luminescent $\mathrm{Au}$ NPs. Reprinted with permission from ref. 164. Copyright (2013) Wiley- $\mathrm{VCH}$.

workers compared the in vivo fate and toxicity of Au NCs functionalized with GSH and BSA (Fig. 11a). ${ }^{160}$ They found that $90 \%$ of $\mathrm{Au}$ from GSH-Au NCs can be cleared through the renal clearance after 28 days, while only less than 5\% of $\mathrm{Au}$ from BSA-Au NCs can be cleared in the same time period. The difference could be ascribed to the less stability of BSA-Au NCs than GSH-Au NCs in serum, resulting in the formation of aggregated BSA-Au NCs that could be absorbed by the RES organs like liver and spleen. Considering the benefits from the PEGylation of NPs, such as the reduced nonspecific accumulation and prolonged blood circulation, Zheng and coworkers showed that the PEG-functionalized Au NPs (PEG-Au NPs) demonstrated an efficient renal clearance and low RES accumulation (Fig. 11b). ${ }^{164}$ PEG-Au NPs also showed an enhanced tumor targeting than GSH-Au NPs, since the blood distribution half-life of PEG-Au NPs was an order of magnitude longer than that of the GSH-Au NPs. As revealed by these studies, the in vivo toxicity study is quite different and more complicated than the in vitro cytotoxicity study. The development of a suitable and generic functionalization strategy for metal NCs is still a challenge to further improve tumor targeting and renal clearance of the as-designed functional metal NCs.

\section{Summary and perspective}

In summary, we have attempted to give a brief overview on the recent achievements in the functionalization of metal NCs and their related biomedical applications. Owing to the unique properties of metal NCs including their attractive physical and chemical properties and good biocompatibility, ${ }^{165-167}$ the recent rapid developments in the synthesis and surface functionalization of metal NCs have enabled the exploitation of these functionalized NCs for biomedical applications. In one way, these designable surface functionalization strategies can adjust their properties such as stability in solution, strong luminescence, as well as biodistribution and toxicity in the biological set-up, which in turn can empower these functional metal NCs with desirable targeting, imaging, and therapeutic ability for biomedical applications.

Although the surface functionalization of metal NCs has been extensively studied, and it has contributed significantly to push forward the biomedical applications of metal NCs in the past decade, there are still many aspects that should be understood in future. Since the physical and chemical properties of metal NCs could be obviously affected by their surface chemistry, it is desirable to have a deeper fundamental understanding on the principles about how to tailor metal NCs with desired properties, such as stability, structure, and luminescence. Moreover, it remains a challenge to precisely incorporate functional metal NCs with a certain number of functional ligands (or controllable functionalities on the NC surface). To fulfil the practical need and translate metal NCs into clinical practice, more systematic studies should be carried out to figure out the effects of functionalities of metal NCs on their targeting, biodistribution, and toxicity in the complicated biological set-up. By integrating the intrinsic luminescence of metal NCs with other imaging and therapeutic agents, multifunctional metal NC-based nanocomposites would be achieved for multi-modal imaging guided therapy. We can anticipate that the functionalized metal NCs would serve as an important platform in the area of biomedical applications.

\section{Acknowledgements}

The authors acknowledge the financial support from the Ministry of Education, Singapore, under Grant No. R-279-000409-112.

\section{References}

1 R. Jin, Nanoscale, 2015, 7, 1549.

2 C. M. Aikens, J. Phys. Chem. Lett., 2011, 2, 99.

3 A. Desireddy, B. E. Conn, J. Guo, B. Yoon, R. N. Barnett, B. M. Monahan, K. Kirschbaum, W. P. Griffith, R. L. Whetten, U. Landman and T. P. Bigioni, Nature, 2013, 501, 399.

4 R. W. Murray, Chem. Rev., 2008, 108, 2688.

5 Z. Luo, K. Zheng and J. Xie, Chem. Commun., 2014, 50, 5143.

6 B. Han and E. Wang, Anal. Bioanal. Chem., 2012, 402, 129.

7 N. Goswami, K. Zheng and J. Xie, Nanoscale, 2014, 6, 13328.

8 Z. Wu and R. Jin, Nano Lett., 2010, 10, 2568.

9 T. D. Green, C. Yi, C. Zeng, R. Jin, S. McGill and K. L. Knappenberger, J. Phys. Chem. A, 2014, 118, 10611.

10 W. Kurashige, M. Yamaguchi, K. Nobusada and Y. Negishi, J. Phys. Chem. Lett., 2012, 3, 2649.

11 C. Y. Tay, Y. Yu, M. I. Setyawati, J. Xie and D. T. Leong, Nano Res., 2014, 7, 805.

12 X. Yuan, N. Goswami, I. Mathews, Y. Yu and J. Xie, Nano Res., 2015, 8, 3488. 
13 L.-Y. Chen, C.-W. Wang, Z. Yuan and H.-T. Chang, Anal. Chem., 2015, 87, 216.

14 L. Shang, S. Dong and G. U. Nienhaus, Nano Today, 2011, 6, 401.

15 S. Knoppe and T. Bürgi, Acc. Chem. Res., 2014, 47, 1318.

16 C. D. Bain, E. B. Troughton, Y. T. Tao, J. Evall, G. M. Whitesides and R. G. Nuzzo, J. Am. Chem. Soc., 1989, 111, 321.

17 H. Häkkinen, Nat. Chem., 2012, 4, 443.

18 C. J. Ackerson, P. D. Jadzinsky and R. D. Kornberg, J. Am. Chem. Soc., 2005, 127, 6550.

19 T. G. Schaaff, G. Knight, M. N. Shafigullin, R. F. Borkman and R. L. Whetten, J. Phys. Chem. B, 1998, 102, 10643.

20 M. J. Hostetler, J. E. Wingate, C.-J. Zhong, J. E. Harris, R. W. Vachet, M. R. Clark, J. D. Londono, S. J. Green, J. J. Stokes, G. D. Wignall, G. L. Glish, M. D. Porter, N. D. Evans and R. W. Murray, Langmuir, 1998, 14, 17.

21 X. Yuan, B. Zhang, Z. Luo, Q. Yao, D. T. Leong, N. Yan and J. Xie, Angew. Chem., Int. Ed., 2014, 53, 4623.

22 X. Yuan, N. Goswami, W. Chen, Q. Yao and J. Xie, Chem. Commun., 2016, 52, 5234.

23 F. Aldeek, M. A. Muhammed, G. Palui, N. Zhan and H. Mattoussi, ACS Nano, 2013, 7, 2509.

24 Z. Wu, E. Lanni, W. Chen, M. E. Bier, D. Ly and R. Jin, J. Am. Chem. Soc., 2009, 131, 16672.

25 Z. Tang, B. Xu, B. Wu, M. W. Germann and G. Wang, J. Am. Chem. Soc., 2010, 132, 3367.

26 Z. Tang, D. A. Robinson, N. Bokossa, B. Xu, S. Wang and G. Wang, J. Am. Chem. Soc., 2011, 133, 16037.

27 H. Yang, Y. Wang, H. Huang, L. Gell, L. Lehtovaara, S. Malola, H. Häkkinen and N. Zheng, Nat. Commun., 2013, 4, 2422.

28 X.-K. Wan, S.-F. Yuan, Q. Tang, D.-E. Jiang and Q.-M. Wang, Angew. Chem., Int. Ed., 2015, 54, 5977.

29 S. Y. Park, A. K. Lytton-Jean, B. Lee, S. Weigand, G. C. Schatz and C. A. Mirkin, Nature, 2008, 451, 553.

30 J. T. Petty, J. Zheng, N. V. Hud and R. M. Dickson, J. Am. Chem. Soc., 2004, 126, 5207.

31 J. Liu, TrAC, Trends Anal. Chem., 2014, 58, 99.

32 T. A. C. Kennedy, J. L. MacLean and J. Liu, Chem. Commun., 2012, 48, 6845.

33 G. Liu, Y. Shao, K. Ma, Q. Cui, F. Wu and S. Xu, Gold Bull., 2012, 45, 69.

34 G. Liu, Y. Shao, J. Peng, W. Dai, L. Liu, S. Xu, F. Wu and X. Wu, Nanotechnology, 2013, 24, 345502.

35 Z. Qing, X. He, D. He, K. Wang, F. Xu, T. Qing and X. Yang, Angew. Chem., Int. Ed., 2013, 52, 9719.

36 A. Rotaru, S. Dutta, E. Jentzsch, K. Gothelf and A. Mokhir, Angew. Chem., Int. Ed., 2010, 49, 5665.

37 J. Xie, Y. Zheng and J. Y. Ying, J. Am. Chem. Soc., 2009, 131, 888.

38 S.-K. Sun, L.-X. Dong, Y. Cao, H.-R. Sun and X.-P. Yan, Anal. Chem., 2013, 85, 8436.

39 J. Xie, Y. Zheng and J. Y. Ying, Chem. Commun., 2010, 46, 961.

40 Y.-H. Lin and W.-L. Tseng, Anal. Chem., 2010, 82, 9194.
41 F. Wen, Y. Dong, L. Feng, S. Wang, S. Zhang and X. Zhang, Anal. Chem., 2011, 83, 1193.

42 C.-L. Liu, H.-T. Wu, Y.-H. Hsiao, C.-W. Lai, C.-W. Shih, Y.-K. Peng, K.-C. Tang, H.-W. Chang, Y.-C. Chien, J.-K. Hsiao, J.-T. Cheng and P.-T. Chou, Angew. Chem., Int. Ed., 2011, 50, 7056.

43 X. Xia, Y. Long and J. Wang, Anal. Chim. Acta, 2013, 772, 81.

44 L.-L. Wang, J. Qiao, L. Qi, X.-Z. Xu and D. Li, Sci. China: Chem., 2015, 58, 1508.

45 N. Goswami, A. Giri, M. S. Bootharaju, P. L. Xavier, T. Pradeep and S. K. Pal, Anal. Chem., 2011, 83, 9676.

46 C. Guo and J. Irudayaraj, Anal. Chem., 2011, 83, 2883.

47 S. Kumar, M. D. Bolan and T. P. Bigioni, J. Am. Chem. Soc., 2010, 132, 13141.

48 Y. Negishi, K. Nobusada and T. Tsukuda, J. Am. Chem. Soc., 2005, 127, 5261.

49 X. Yuan, Z. Luo, Q. Zhang, X. Zhang, Y. Zheng, J. Y. Lee and J. Xie, ACS Nano, 2011, 5, 8800.

50 Z. Luo, X. Yuan, Y. Yu, Q. Zhang, D. T. Leong, J. Y. Lee and J. Xie, J. Am. Chem. Soc., 2012, 134, 16662.

51 Q. Wen, Y. Gu, L.-J. Tang, R.-Q. Yu and J.-H. Jiang, Anal. Chem., 2013, 85, 11681.

52 S. Lee, J. Xie and X. Chen, Biochemistry, 2010, 49, 1364.

53 J. Zheng, J. T. Petty and R. M. Dickson, J. Am. Chem. Soc., 2003, 125, 7780.

54 J. Zheng and R. M. Dickson, J. Am. Chem. Soc., 2002, 124, 13982.

55 J. Li, W. Wang, D. Sun, J. Chen, P.-H. Zhang, J.-R. Zhang, Q. Min and J.-J. Zhu, Chem. Sci., 2013, 4, 3514.

56 G. Li and Y. Luo, Inorg. Chem., 2008, 47, 360.

57 R. W. Scott, O. M. Wilson, S. K. Oh, E. A. Kenik and R. M. Crooks, J. Am. Chem. Soc., 2004, 126, 15583.

58 I. Díez, M. Pusa, S. Kulmala, H. Jiang, A. Walther, A. S. Goldmann, A. H. E. Müller, O. Ikkala and R. H. A. Ras, Angew. Chem., Int. Ed., 2009, 48, 2122.

59 S. Liu, F. Lu and J.-J. Zhu, Chem. Commun., 2011, 47, 2661.

60 H. Duan and S. Nie, J. Am. Chem. Soc., 2007, 129, 2412.

61 F. Qu, N. B. Li and H. Q. Luo, Anal. Chem., 2012, 84, 10373.

62 F. Qu, N. B. Li and H. Q. Luo, Langmuir, 2013, 29, 1199.

63 B. Santiago González, M. J. Rodríguez, C. Blanco, J. Rivas, M. A. López-Quintela and J. M. G. Martinho, Nano Lett., 2010, 10, 4217.

64 Y. Ling, N. Zhang, F. Qu, T. Wen, Z. F. Gao, N. B. Li and H. Q. Luo, Spectrochim. Acta, Part A, 2014, 118, 315.

65 L. V. Nair, S. S. Nazeer, R. S. Jayasree and A. Ajayaghosh, ACS Nano, 2015, 9, 5825.

66 V. Marjomäki, T. Lahtinen, M. Martikainen, J. Koivisto, S. Malola, K. Salorinne, M. Pettersson and H. Häkkinen, Proc. Natl. Acad. Sci. U. S. A., 2014, 111, 1277.

67 R. Ban, E. S. Abdel-Halim, J. Zhang and J.-J. Zhu, Analyst, 2015, 140, 1046.

68 F. Dubois, B. Mahler, B. Dubertret, E. Doris and C. Mioskowski, J. Am. Chem. Soc., 2007, 129, 482.

69 R. Sardar, A. M. Funston, P. Mulvaney and R. W. Murray, Langmuir, 2009, 25, 13840. 
70 M. J. Hostetler, A. C. Templeton and R. W. Murray, Langmuir, 1999, 15, 3782.

71 A. C. Templeton, M. P. Wuelfing and R. W. Murray, Acc. Chem. Res., 2000, 33, 27.

72 L. G. AbdulHalim, N. Kothalawala, L. Sinatra, A. Dass and O. M. Bakr, J. Am. Chem. Soc., 2014, 136, 15865.

73 S. Knoppe, R. Azoulay, A. Dass and T. Bürgi, J. Am. Chem. Soc., 2012, 134, 20302.

74 Y. Shichibu, Y. Negishi, T. Tsukuda and T. Teranishi, J. Am. Chem. Soc., 2005, 127, 13464.

75 G. H. Woehrle, L. O. Brown and J. E. Hutchison, J. Am. Chem. Soc., 2005, 127, 2172.

76 E. S. Shibu, M. A. H. Muhammed, T. Tsukuda and T. Pradeep, J. Phys. Chem. C, 2008, 112, 12168.

77 A. Fernando and C. M. Aikens, J. Phys. Chem. C, 2015, 119, 20179.

78 M. S. Bootharaju, V. M. Burlakov, T. M. D. Besong, C. P. Joshi, L. G. AbdulHalim, D. M. Black, R. L. Whetten, A. Goriely and O. M. Bakr, Chem. Mater., 2015, 27, 4289.

79 S.-I. Tanaka, J. Miyazaki, D. K. Tiwari, T. Jin and Y. Inouye, Angew. Chem., Int. Ed., 2011, 50, 431.

80 S.-Y. Lin, N.-T. Chen, S.-P. Sum, L.-W. Lo and C.-S. Yang, Chem. Commun., 2008, 4762.

81 C.-A. J. Lin, T.-Y. Yang, C.-H. Lee, S. H. Huang, R. A. Sperling, M. Zanella, J. K. Li, J.-L. Shen, H.-H. Wang, H.-I. Yeh, W. J. Parak and W. H. Chang, ACS Nano, 2009, 3, 395.

82 C.-C. Huang, C.-K. Chiang, Z.-H. Lin, K.-H. Lee and H.-T. Chang, Anal. Chem., 2008, 80, 1497.

83 Q. Yao, X. Yuan, Y. Yu, Y. Yu, J. Xie and J. Y. Lee, J. Am. Chem. Soc., 2015, 137, 2128.

84 K. Pyo, V. D. Thanthirige, K. Kwak, P. Pandurangan, G. Ramakrishna and D. Lee, J. Am. Chem. Soc., 2015, 137, 8244.

85 K. Yang, L. Feng, X. Shi and Z. Liu, Chem. Soc. Rev., 2013, 42, 530 .

86 C. Wang, J. Li, C. Amatore, Y. Chen, H. Jiang and X.-M. Wang, Angew. Chem., Int. Ed., 2011, 50, 11644.

87 A. Chandrasekar and T. Pradeep, J. Phys. Chem. C, 2012, 116, 14057.

88 X. Liu, F. Wang, R. Aizen, O. Yehezkeli and I. Willner, J. Am. Chem. Soc., 2013, 135, 11832.

89 Y. Wang, J.-T. Chen and X.-P. Yan, Anal. Chem., 2013, 85, 2529.

90 Y. Tao, Y. Lin, Z. Huang, J. Ren and X. Qu, Adv. Mater., 2013, 25, 2594.

91 H. Yin, H. Tang, D. Wang, Y. Gao and Z. Tang, ACS Nano, 2012, 6, 8288.

92 P. Zhao, K. He, Y. Han, Z. Zhang, M. Yu, H. Wang, Y. Huang, Z. Nie and S. Yao, Anal. Chem., 2015, 87, 9998.

93 L.-X. Zhang, J.-L. Shi, J. Yu, Z.-L. Hua, X.-G. Zhao and M.-L. Ruan, Adv. Mater., 2002, 14, 1510.

94 J. N. Tiwari, K. Nath, S. Kumar, R. N. Tiwari, K. C. Kemp, N. H. Le, D. H. Youn, J. S. Lee and K. S. Kim, Nat. Commun., 2013, 4, 2221.

95 G. Wang, G. Xu, Y. Zhu and X. Zhang, Chem. Commun., 2014, 50, 747.
96 A. V. Ellis, K. Vjayamohanan, R. Goswaimi, N. Chakrapani, L. S. Ramanathan, P. M. Ajayan and G. Ramanath, Nano Lett., 2003, 3, 279.

97 H. Huang, Z. He, X. Lin, W. Ruan, Y. Liu and Z. Yang, Appl. Catal., A, 2015, 490, 65.

98 N. Erathodiyil and J. Y. Ying, Acc. Chem. Res., 2011, 44, 925.

99 X. Wu, L. Li, L. Zhang, T. Wang, C. Wang and Z. Su, J. Mater. Chem. B, 2015, 3, 2421.

100 X. Wu, C. X. Li, S. Liao, L. Li, T. Wang, Z. Su, C. Wang, J. Zhao, C. Sui and J. Lin, Chem. - Eur. J., 2014, 20, 8876.

101 P. Huang, J. Lin, S. Wang, Z. Zhou, Z. Li, Z. Wang, C. Zhang, X. Yue, G. Niu, M. Yang, D. Cui and X. Chen, Biomaterials, 2013, 34, 4643.

102 X. L. Guével, B. Hötzer, G. Jung and M. Schneider, J. Mater. Chem., 2011, 21, 2974.

103 Y. Wu, J. Huang, T. Zhou, M. Rong, Y. Jiang and X. Chen, Analyst, 2013, 138, 5563.

104 L. Qin, X. He, L. Chen and Y. Zhang, ACS Appl. Mater. Interfaces, 2015, 7, 5965.

105 V. Subramanian, E. E. Wolf and P. V. Kamat, J. Am. Chem. Soc., 2004, 126, 4943.

106 L. L. Chng, N. Erathodiyil and J. Y. Ying, Acc. Chem. Res., 2013, 46, 1825.

107 Y.-S. Chen and P. V. Kamat, J. Am. Chem. Soc., 2014, 136, 6075.

108 J.-J. Liu, X.-R. Song, Y.-W. Wang, A.-X. Zheng, G.-N. Chen and H.-H. Yang, Anal. Chim. Acta, 2012, 749, 70.

109 J. Yin, X. He, K. Wang, F. Xu, J. Shangguan, D. He and H. Shi, Anal. Chem., 2013, 85, 12011.

110 H.-C. Yeh, J. Sharma, J. J. Han, J. S. Martinez and J. H. Werner, Nano Lett., 2010, 10, 3106.

111 J. Li, X. Zhong, H. Zhang, X. C. Le and J.-J. Zhu, Anal. Chem., 2012, 84, 5170.

112 L. Zhang, J. Zhu, S. Guo, T. Li, J. Li and E. Wang, J. Am. Chem. Soc., 2013, 135, 2403.

113 J. Li, X. Zhong, F. Cheng, J.-R. Zhang, L.-P. Jiang and J.-J. Zhu, Anal. Chem., 2012, 84, 4140.

114 A. Retnakumari, S. Setua, D. Menon, P. Ravindran, H. Muhammed, T. Pradeep, S. Nair and M. Koyakutty, Nanotechnology, 2010, 21, 055103.

115 Y. Wang, J. Chen and J. Irudayaraj, ACS Nano, 2011, 5, 9718.

116 J. Qiao, X. Mu, L. Qi, J. Deng and L. Mao, Chem. Commun., 2013, 49, 8030.

117 D. An, J. Su, J. K. Weber, X. Gao, R. Zhou and J. Li, J. Am. Chem. Soc., 2015, 137, 8412.

118 C. Sun, Y. Yuan, Z. Xu, T. Ji, Y. Tian, S. Wu, J. Lei, J. Li, N. Gao and G. Nie, Bioconjugate Chem., 2015, 26, 193.

119 C. Sun, H. Yang, Y. Yuan, X. Tian, L. Wang, Y. Guo, L. Xu, J. Lei, N. Gao, G. J. Anderson, X.-J. Liang, C. Chen, Y. Zhao and G. Nie, J. Am. Chem. Soc., 2011, 133, 8617.

120 R. Vankayala, C.-L. Kuo, K. Nuthalapati, C.-S. Chiang and K. C. Hwang, Adv. Funct. Mater., 2015, 25, 5934.

121 C. Zhang, Z. Zhou, Q. Qian, G. Gao, C. Li, L. Feng, Q. Wang and D. Cui, J. Mater. Chem. B, 2013, 1, 5045.

122 C. Zhou, M. Long, Y. Qin, X. Sun and J. Zheng, Angew. Chem., Int. Ed., 2011, 50, 3168. 
123 L.-S. Lin, X. Yang, Z.-S. Cong, J.-B. Cao, K.-M. Ke, Q.-L. Peng, J. Gao, H.-H. Yang, G. Liu and X. Chen, ACS Nano, 2014, 8, 3876.

124 X.-R. Song, S.-X. Yu, G.-X. Jin, X. Wang, J. Chen, J. Li, G. Liu and H.-H. Yang, Small, 2016, 12, 1506.

125 D.-H. Hu, Z.-H. Sheng, P.-F. Zhang, D.-Z. Yang, S.-H. Liu, P. Gong, D.-Y. Gao, S.-T. Fang, Y.-F. Ma and L.-T. Cai, Nanoscale, 2013, 5, 1624.

126 G. Liang, D. Ye, X. Zhang, F. Dong, H. Chen, S. Zhang, J. Li, X. Shen and J. Kong, J. Mater. Chem. B, 2013, 1, 3545.

127 C. Wang, Y. Yao and Q. Song, J. Mater. Chem. C, 2015, 3, 5910.

128 H. Hu, P. Huang, O. J. Weiss, X. Yan, X. Yue, M. G. Zhang, Y. Tang, L. Nie, Y. Ma, G. Niu, K. Wu and X. Chen, Biomaterials, 2014, 35, 9868.

129 J. Li, J. You, Y. Dai, M. Shi, C. Han and K. Xu, Anal. Chem., 2014, 86, 11306.

130 F. Gao, P. Cai, W. Yang, J. Xue, L. Gao, R. Liu, Y. Wang, Y. Zhao, X. He, L. Zhao, G. Huang, F. Wu, Y. Zhao, Z. Chai and X. Gao, ACS Nano, 2015, 9, 4976.

131 K. T. Butterworth, S. J. McMahon, F. J. Currellab and K. M. Prise, Nanoscale, 2012, 4, 4830.

132 X.-D. Zhang, Z. Luo, J. Chen, S. Song, X. Yuan, X. Shen, H. Wang, Y. Sun, K. Gao, L. Zhang, S. Fan, D. T. Leong, M. Guo and J. Xie, Sci. Rep., 2015, 5, 8669.

133 X.-D. Zhang, Z. Luo, J. Chen, X. Shen, S. Song, Y. Sun, S. Fan, F. Fan, D. T. Leong and J. Xie, Adv. Mater., 2014, 26, 4565 .

134 X.-D. Zhang, J. Chen, Z. Luo, D. Wu, X. Shen, S.-S. Song, Y.-M. Sun, P.-X. Liu, J. Zhao, S. Huo, S. Fan, F. Fan, X.-J. Liang and J. Xie, Adv. Healthcare Mater., 2014, 3, 133.

135 L.-S. Lin, Z.-X. Cong, J. Li, K.-M. Ke, S.-S. Guo, H.-H. Yang and G.-N. Chen, J. Mater. Chem. B, 2014, 2, 1031.

136 M. Sakamoto, T. Tachikawa, M. Fujitsuka and T. Majima, Langmuir, 2009, 25, 13888.

137 T. Das, P. Ghosh, M. S. Shanavas, A. Maity, S. Mondal and P. Purkayastha, Nanoscale, 2012, 4, 6018.

138 E. S. Shibu, S. Sugino, K. Ono, H. Saito, A. Nishioka, S. Yamamura, M. Sawada, Y. Nosaka and V. Biju, Angew. Chem., Int. Ed., 2013, 52, 10559.

139 H. Kawasaki, S. Kumar, G. Li, C. Zeng, D. R. Kauffman, J. Yoshimoto, Y. Iwasaki and R. Jin, Chem. Mater., 2014, 26, 2777.

140 P. Zhang, Y. Chen, Y. Zeng, C. Shen, R. Li, Z. Guo, S. Li, Q. Zheng, C. Chu, Z. Wang, Z. Zheng, R. Tian, S. Ge, X. Zhang, N.-S. Xia, G. Liu and X. Chen, Proc. Natl. Acad. Sci. U. S. A., 2015, 112, 6129.

141 J. Li, C. Zheng, S. Cansiz, C. Wu, J. Xu, C. Cui, Y. Liu, W. Hou, Y. Wang, I. Teng, H.-H. Yang and W. Tan, J. Am. Chem. Soc., 2015, 137, 1412.

142 X.-R. Song, X. Wang, S.-X. Yu, J. Cao, S.-H. Li, J. Li, G. Liu, H.-H. Yang and X. Chen, Adv. Mater., 2015, 27, 3285.

143 T. Chen, S. Xu, T. Zhao, L. Zhu, D. Wei, Y. Li, H. Zhang and C. Zhao, ACS Appl. Mater. Interfaces, 2012, 4, 5766.

144 Y. Tao, Z. Li, E. Ju, J. Ren and X. Qu, Nanoscale, 2013, 5, 6154.
145 N. Li, Y. Chen, Y.-M. Zhang, Y. Yang, Y. Su, J.-T. Chen and Y. Liu, Sci. Rep., 2014, 4, 4164.

146 Y. Tao, E. Ju, Z. Li, J. Ren and X. Qu, Adv. Funct. Mater., 2014, 24, 1004.

147 D. Chen, Z. Luo, N. Li, J. Y. Lee, J. Xie and J. Lu, Adv. Funct. Mater., 2013, 23, 4324.

148 C. Zhang, C. Li, Y. Liu, J. Zhang, C. Bao, S. Liang, Q. Wang, Y. Yang, H. Fu, K. Wang and D. Cui, Adv. Funct. Mater., 2015, 25, 1314.

149 C. Zhang, Z. Zhou, X. Zhi, Y. Ma, K. Wang, Y. Wang, Y. Zhang, H. Fu, W. Jin, F. Pan and D. Cui, Theranostics, 2015, 5, 134.

150 L. Yang, L. Shang and G. U. Nienhaus, Nanoscale, 2013, 5, 1537.

151 J.-Y. Zhao, R. Cui, Z.-L. Zhang, M. Zhang, Z.-X. Xie and D.-W. Pang, Nanoscale, 2014, 6, 13126.

152 L. Dong, M. Li, S. Zhang, J. Li, G. Shen, Y. Tu, J. Zhu and J. Tao, Small, 2015, 11, 2571.

153 T. D. Fernández, J. R. Pearson, M. P. Leal, M. J. Torres, M. Blanca, C. Mayorga and X. L. Guével, Biomaterials, 2015, 43, 1

154 H. S. Choi, W. Liu, P. Misra, E. Tanaka, J. P. Zimmer, B. I. Ipe, M. G. Bawendi and J. V. Frangioni, Nat. Biotechnol., 2007, 25, 1165.

155 J. Liu, M. Yu, C. Zhou, S. Yang, X. Ning and J. Zheng, J. Am. Chem. Soc., 2013, 135, 4978.

156 J. Liu, M. Yu, C. Zhou and J. Zheng, Mater. Today, 2013, 16, 477 .

157 M. Zhou, J. Li, S. Liang, A. K. Sood, D. Liang and C. Li, ACS Nano, 2015, 9, 7085.

158 F. Liu, X. He, H. Chen, J. Zhang, H. Zhang and Z. Wang, Nat. Commun., 2015, 6, 8003.

159 H. Chen, G. D. Wang, W. Tang, T. Todd, Z. Zhen, C. Tsang, K. Hekmatyar, T. Cowger, R. B. Hubbard, W. Zhang, J. Stickney, B. Shen and J. Xie, Adv. Mater., 2014, 26, 6761.

160 X.-D. Zhang, D. Wu, X. Shen, P.-X. Liu, F.-Y. Fan and S.-J. Fan, Biomaterials, 2012, 33, 4628.

161 X.-D. Zhang, Z. Luo, J. Chen, H. Wang, S.-S. Song, X. Shen, W. Long, Y.-M. Sun, S. Fan, K. Zheng, D. T. Leong and J. Xie, Small, 2015, 11, 1683.

162 O. A. Wong, R. J. Hansen, T. W. Ni, C. L. Heinecke, W. S. Compel, D. L. Gustafson and C. J. Ackerson, Nanoscale, 2013, 5, 10525.

163 X.-D. Zhang, J. Yang, S.-S. Song, W. Long, J. Chen, X. Shen, H. Wang, Y.-M. Sun, P.-X. Liu and S. Fan, Int. J. Nanomed., 2014, 9, 2069.

164 J. Liu, M. Yu, X. Ning, C. Zhou, S. Yang and J. Zheng, Angew. Chem., Int. Ed., 2013, 52, 12572.

165 W. Wei, Y. Lu, W. Chen and S. Chen, J. Am. Chem. Soc., 2011, 133, 2060

166 S. Wang, X. Meng, A. Das, T. Li, Y. Song, T. Cao, X. Zhu, M. Zhu and R. Jin, Angew. Chem., Int. Ed., 2014, 53, 2376.

167 S. Choi, R. M. Dickson and J. Yu, Chem. Soc. Rev., 2012, 41, 1867. 